\title{
Microchannelled chitosan sponge grafting with hydrophobic alkyl chain for enhanced noncompressible hemorrhage and tissue regeneration
}

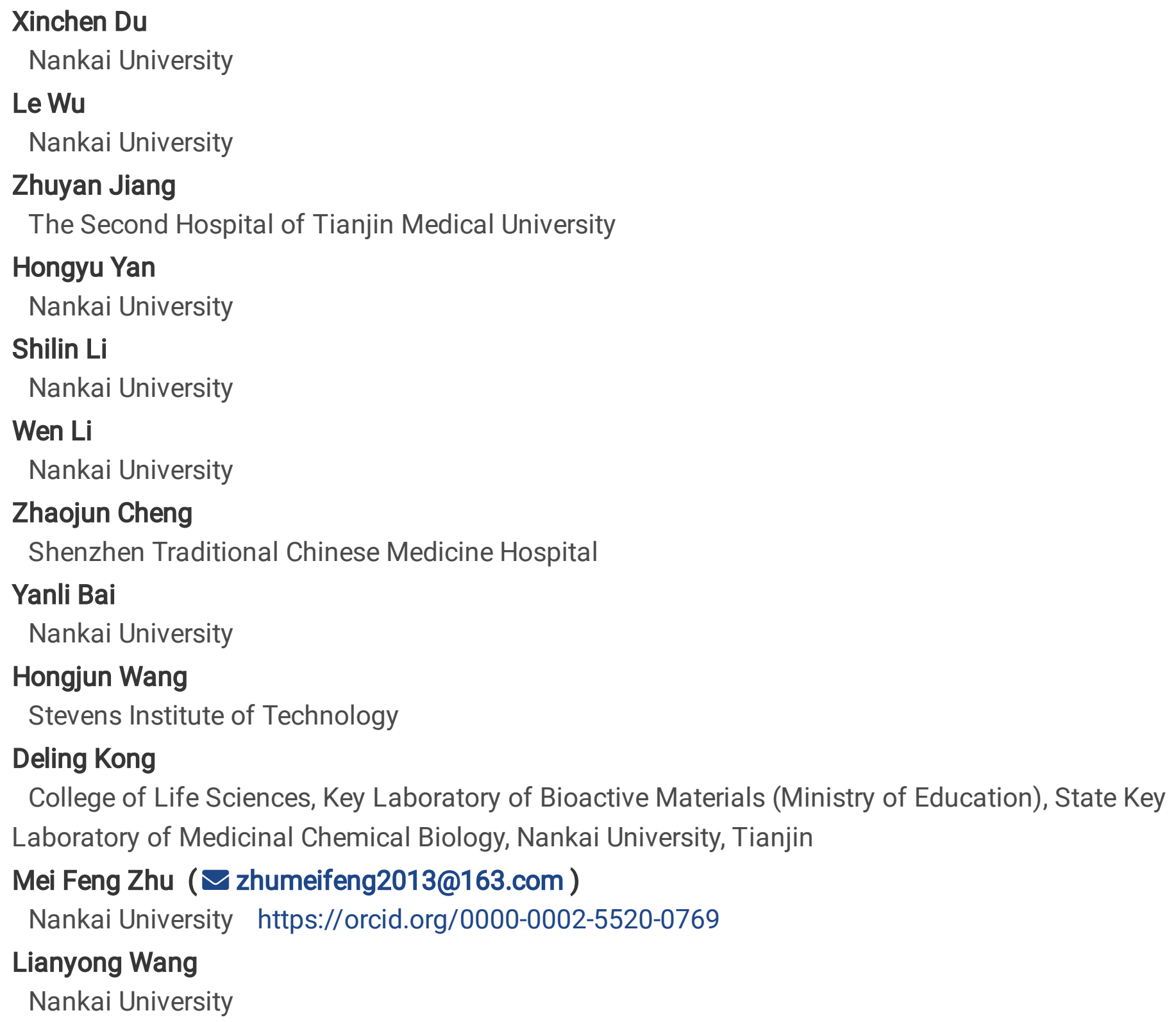

Article 
Keywords: Shape memory chitosan sponge, microchannel, noncompressible hemorrhage, in situ tissue regeneration

Posted Date: November 30th, 2020

DOI: https://doi.org/10.21203/rs.3.rs-109926/v1

License: (1) This work is licensed under a Creative Commons Attribution 4.0 International License. Read Full License

Version of Record: A version of this preprint was published at Nature Communications on August 5th, 2021. See the published version at https://doi.org/10.1038/s41467-021-24972-2. 


\section{Abstract}

Developing an anti-infective shape-memory hemostatic sponge with ability of guiding in situ tissue regeneration for noncompressible hemorrhage in civilian and battlefield settings remains a challenge. Here, hemostatic chitosan sponge with highly interconnective microchannels was engineered by combining 3D printed fiber leaching and freeze-drying methods and then modified with hydrophobic alkyl chains. The microchannelled alkylated chitosan sponge (MACS) exhibited a strong capacity for water/blood absorption and rapid shape recovery. Compared to clinically used gauze, gelatin sponge, CELOX, and CELOX-gauze, the MACS demonstrated higher pro-coagulant and hemostatic capacities in lethally normal/heparinized rat and pig liver perforation models. Also, it exhibited strong anti-infective activity against $S$. aureus and $E$. coli. Additionally, it promoted liver parenchymal cell infiltration, vascularization, and tissue integration in a rat liver defect model. Overall, the MACS demonstrated promising clinical translational potential in cost effectively treating lethal noncompressible hemorrhage and in facilitating wound healing.

\section{Introduction}

Hypotension and multi-organ failure caused by massive blood loss often results in high mortality in civilian and military populations ${ }^{1,2}$. So, rapid and efficient hemorrhage control is of paramount importance in such scenarios. The body's natural coagulation cascade process is activated in response to bleeding, but, incapable of timely stopping severe hemorrhage from a deep and noncompressible perforation wound in the absence of shape-memory hemostats ${ }^{3,4}$. Thus, the development of shapememory hemostats is urgently needed. In general, ideal shape-memory hemostats should possess several properties, including a highly interconnected porous structure, active coagulation, strong antiinfection activity, biocompatibility, biodegradability, ready availability, low weight, and low $\operatorname{cost}^{4}, 5,6,7$. Notably, an interconnected porous structure permits fluid to flow freely in and out of hemostats, which allows the hemostats to be fixed by draining off the free water and promotes fast recovery to their initial shapes by absorbing the fluid ${ }^{6}$. Rapid-shape recovery timely exerts pressure on the wound, leading to effective hemorrhage control ${ }^{6,8}$. Moreover, hemostats left in the injury site and used in directly guided in situ tissue regeneration are more practical for clinical application ${ }^{9}$.

Until now, several shape-memory hemostats have been developed, and some have been applied in clinical practice $^{10,11,12,13}$. For instance, the XStat ${ }^{\mathrm{TM}}$ device composed of multiple compressed cellulose sponges was shown to rapidly expand to fill and exert pressure on a wound to control hemorrhage ${ }^{10}$. However, it took much more time to take out each sponge from the wound bed due to its nondegradable property, which may cause patient discomfort ${ }^{8}$. Moreover, such sponge lacking highly interconnected porous structure was incapable of guiding tissue repair. Many shape-memory polymer foams as hemostats have been applied to treat noncompressible hemorrhage and exhibited a certain degree of hemostatic ability ${ }^{11}$, 12, 13. However, they displayed limited absorption of blood and required decades of seconds to restore their shapes, which may cause the prologation of hemostasis time and more blood loss ${ }^{5}$. Injectable 
cryogels with high blood absorbability and rapid-shape recovery capacity have also been developed for treatment of noncompressible hemorrhage ${ }^{6,14,15}$. The hemostatic effect of these materials was achieved by restoring shape and applying mechanical compression on the wound. Shape-recovery property mainly originates from the reversible change of porous structure ${ }^{10,11,12,13}$. However, the pores inside these hemostats generated by gas foaming or ice crystal removing methods possess low interconnectivity, which might slow down the blood flow into hemostats, resulting in weakened hemostatic efficiency. The effect of pore structure, especially interconnectivity, on hemostatic performance was usually ignored in the design and construction of hemostats $5,8,10,14$. Besides, some of these hemostats lacked strong active pro-coagulant and anti-infective properties, which may result in their failure to complete the hemostasis in a timely and effective way and in their inability to protect wounds from bacterial infection. Therefore, simultaneously regulating pore structure and active modification is expected to improve the hemostatic and anti-infective effects of these hemostats.

Incorporating a microchannel into three-dimensional (3D) constructs is a simple and controllable architectural feature, and capable of promoting transport of nutrients, oxygen, and metabolites, host cell infiltration, vascularization, and integration with the surrounding tissue $16,17,18,19$. To create an embedded and hollow microchannel, the sacrificial fibrous template with a well-defined 3D architecture was first enclosed within a matrix material solution and later removed via external stimuli ${ }^{20}$. Such an approach showed better controllability and interconnectivity in pore structure than conventional pore-forming methods, including gas foaming and ice crystal removing ${ }^{18}$. Still, developing shape-memory hemostats with a microchannel structure has not been previously investigated.

Chitosan (CS) has been used to prepare hemostats due to its inherent properties, such as biocompatibility, biodegradability, non-toxicity, anti-infection ability, hemostasis, and so forth ${ }^{21,22}$. Nevertheless, as mentioned above, its hemostatic and anti-infective properties were limited, especially in cases complicated by severe hemorrhage and bacterial infections ${ }^{23}$. Previous studies by our group and others have demonstrated that grafting hydrophobic alkyl chains onto a CS backbone could improve its hemostatic and anti-infective abilities, attributed to the strong hydrophobic interactions between the alkyl chains and the membranes of red blood cells (RBCs), platelets, and bacteria $23,24,25,26$.

Based on these studies, we propose that the shape-memory, pro-coagulant and anti-infective properties of hemostats for noncompressible hemorrhage and in situ tissue regeneration can be improved by optimizing the materials pore structure and further active modification. The CS sponges with microchannels were firstly engineered by combining 3D printing polymer microfiber template leaching and freeze-drying methods. To further improve pro-coagulant and anti-infective properties, the microchannelled CS sponges were modified with hydrophobic alkyl chains, named MACSs. They presented a highly interconnective and controllable microchannel structure, high water/blood absorbability, a fast shape-recovery property, a strong coagulation-promoting effect, and anti-infection activity. Notably, they demonstrated better hemostatic performance compared with clinically used gauze, gelatin sponge, CELOX, and CELOX-gauze in lethally normal/heparinized rat and normal pig liver 
perforation wound models. Moreover, they enabled liver cell infiltration, vascularization, and tissue/sponge integration. These results suggest that the MACSs may be beneficial for treating noncompressible hemorrhage and for promoting in situ penetrating wound healing, and thus, have convincing potential for clinical and translational applications.

\section{Results And Discussion}

\section{Fabrication and characterization of the MACSs}

According to our design criteria, the MACSs were fabricated by the procedure illustrated in Fig. 1A. First, the sacrificial PLA microfiber templates were printed by a 3D printer (Fig. 1B and Supplementary Fig. 1). Then, the templates were lyophilized after filling with a $4 \%(w / v)$ CS solution. A CS sponge with a uniform microchannel structure was obtained following complete removal of the PLA templates, which was confirmed by FTIR measurement (Supplementary Fig. 2). The resultant CS sponge was further grafted with hydrophobic alkyl chains to improve its pro-coagulant and anti-infective properties. The grafting was carried out via a highly efficient Schiff-base reaction between the amine group of CS and aldehyde group of DA (Fig. 2A). The unstable imine bonds $(\mathrm{C}=\mathrm{N})$ were converted into stable alkylamine (C-N) linkages using a reductant $\left(\mathrm{NaCNBH}_{3}\right)$. Compared to the $\mathrm{N} 1 \mathrm{~s}$ spectrum of the $\mathrm{CS}$ sponge, the appearance of C$\mathrm{N}^{*} \mathrm{H}-\mathrm{C}$ with a peak area of $39.84 \%$ and reduction of the peak area of $\mathrm{C}-\mathrm{N}^{*} \mathrm{H}_{2}$ in the $\mathrm{N} 1 \mathrm{~s}$ spectrum of the alkylated CS sponge indicated the successful reaction of the amine and aldehyde groups (Fig. 2B-D). Moreover, the modified CS sponge showed increased Atom Conc \% and Mass Conc \% of C1s, further demonstrating the successful grafting of hydrophobic alkyl chains (Fig. 2E).

Interconnected pores of the hemostatic sponge could endow itself with the ability to concentrate blood clotting factors and rapidly recover initial shape ${ }^{5,10,29}$. Moreover, they were able to provide a comfortable niche to support host cell infiltration, vascularization, and tissue ingrowth ${ }^{30}$. Micro-CT images showed that the alkylated CS sponges with different porosity (MACS-1/2/3) fabricated by a combination of the template leaching method and freeze-drying possessed a uniform microchannel structure with an increased microchannel density (Fig. 1C). The alkylated CS sponge (ACS) prepared by direct freeze-drying presented dense structure. Furthermore, SEM images displayed a hierarchical porous structure including microchannel $(138 \pm 4.3 \mu \mathrm{m})$ and micropores $(8.7 \pm 1.5 \mu \mathrm{m})$ in the MACS-1/2/3 (Fig. 1D-F), while only micropores $(8.4 \pm 0.9 \mu \mathrm{m})$ randomly distributed throughout the ACS. The microchannel structure was highly interconnected and tunable, and distributed uniformly across the MACS-1/2/3 (Supplementary Movies 1-3). However, the micropores distributed in the ACS showed a dense structure and low interconnectivity (Supplementary Movie 4). The interconnectivity of the porous structure played a key role in accelerating hemostasis and guiding tissue regeneration, which usually was ignored in most previous studies $^{5,6,10,13}$. The MACSs were expected to exhibit an obvious advantage in the treatment of noncompressible hemorrhage and in situ tissue regeneration in comparison with reported porous hemostats $5,6,10,14$. Accordingly, the porosity of the MACSs gradually increased from $70 \pm 2.0$ to $90 \pm$ $0.6 \%$ with an increase in filling ratio of PLA microfiber, which were significantly higher than the $31 \pm 0.7 \%$ 
of the ACS (Fig. 1G). Hemostats filled into the wound cavity should possess desirable mechanical strength to prevent their shape deformation caused by external stress from surrounding tissues, thereby providing durable compression on the bleeding site. We first examined the effect of CS concentration on the compressive stress of the MACSs. As the CS concentration increased from 1 to $4 \%(w / v)$, the compressive stress was enhanced from $0.6 \pm 0.2$ to $23 \pm 1.5 \mathrm{kPa}(\mathrm{Fig} .1 \mathrm{H}, \mathrm{I})$. When the CS concentration was lower than $4 \%$, the sponges could not maintain their shapes (Supplementary Fig. 3A). The CS solution with concentration higher than $4 \%$ possessed higher viscosity (Supplementary Fig. 3B, C), and was difficult to be sucked into the gap of the PLA microfiber template under negative pressure. So, the $4 \%$ CS solution was selected to fabricate the MACSs. Next, we investigated the effect of the filling ratio of the PLA microfiber template on the compressive stress. The compressive stress decreased from $46.2 \pm 8.0$ to $8.1 \pm 0.9 \mathrm{kPa}$ by increasing the filling ratio of the PLA microfiber template from 20 to $60 \%$ (Fig. $1 \mathrm{~J}, \mathrm{~K}$ ). Indeed, the compressive stress of the MACSs was significantly lower than the $138.0 \pm 16.3 \mathrm{kPa}$ of the ACS due to the incorporation of the microchannel structure. To better approach practical application, we further detected the compression stress of the sponges after absorbing blood. All the sponges exhibited reinforced mechanical strength (Fig. $1 \mathrm{~L}, \mathrm{M}$ ), attributing to the formation of blood clots within the sponges. Both the CS and hydrophobic alkyl chain have been proven to facilitate blood clotting by promoting the adhesion and activation of platelets and the aggregation of RBCs. The MACSs had a higher mechanically reinforced fold than the ACS (Fig. 1N) ${ }^{1,9}$. Also, the mechanically reinforced fold of the MACSs gradually enhanced with the increase in porosity (Fig. 1N). The MACSs with high porosity and large surface area could absorb more blood and facilitate the blood to fully contact with the matrix to form more blood clots. Also, the alkylated CS sponge (MACS-2) displayed an improved mechanically reinforced fold compared to the unmodified CS sponge (MCS-2) due to the introduction of hydrophobic alkyl chains (Fig. 1N).

Fig. 1 Fabrication and characterization of the MACSs with different porosity. (A) Schematic illustration of the fabrication process of the MACSs. (B) Stereomicroscopic images of the PLA microfiber template, CS/PLA composite, microchannelled CS sponge, and microchannelled alkylated CS sponge. (C, D) MicroCT and SEM images showing the macro and microstructure of the ACS and MACS-1/2/3. (E, F) The pore size of the ACS and MACS-1/2/3 in cross-section and longitudinal-section. (G) The porosity of the ACS and MACS-1/2/3. $(\mathrm{H}, \mathrm{I})$ Compressive stress-strain curves and compressive stress of the MACSs with different CS concentrations $(1,2$, and $4 \%(w / v)) .(J, K, L, M)$ Compressive stress-strain curves and compressive stress of the ACS, MCS-2, and MACS-1/2/3 before and after absorbing blood. (N) Mechanically reinforced folds of the ACS, MCS-2, and MACS-1/2/3 before and after absorbing blood. $n=3$, Data are means \pm SD. ns indicated no significant difference, $* P<0.05, * * P<0.01, * * * P<0.001$.

Fig. 2 Chemical characterization of the MACSs. (A) Modification of the CS sponge with DA in the presence of $\mathrm{NaCNBH}_{3}$ as a reducing agent. (B, C) XPS spectra showing N1s peak of the CS and alkylated CS sponges. (D, E) The area of N1s peak and the calibrate value of C1s in the CS and alkylated CS sponges. 
The main hemostatic mechanism of expandable hemostats was mechanical compression on the bleeding site, which mainly resulted from water/blood-triggered shape recovery and volume expansion ${ }^{1,5}$, $14,27,29$. Thus, strong water/blood absorbability was indispensable for expandable hemostats. After absorbing water and blood, the MACSs rapidly sank to the bottom of the container, while the ACS suspended in water and blood (Fig. 3A, B), revealing that the MACSs could absorb a higher volume of water and blood compared with the ACS. The maximum water and blood absorption capacity of the MACSs was significantly higher than that of the ACS and gradually improved with an increase in the porosity (Fig. 3C-F). Notably, the MACSs took much less time to achieve saturated water/blood absorption than that of the ACS (Fig. 3C, D). The water and blood absorption rate of the MACSs was higher than that of the ACS (Fig. 3G, H), which resulted from the increased number of microchannels. The more microchannels present, the higher the water/blood absorption rate. We further stimulated the fluid absorption behavior of the sponges, whose pore size originated from the statistical analysis of SEM images, as shown in Fig. 3l. We found that the fluid speed in the microchannels of the alkylated sponges (MACS-1/2/3) was higher than that in micropores of the ACS. The higher number of microchannels resulted in a larger area of distribution of the high fluid speed. The total fluid speed of the MACSs was notably higher than that of the ACS and gradually improved as the number of microchannels increased.

Fig. 3 The water/blood absorbability of the ACS and MACSs. (A, B) Photographs of the ACS and MACS$1 / 2 / 3$ after absorbing water and blood. (C, D) Water and blood absorption capacity-time dynamic curves of the ACS and MACS-1/2/3. (E, F) Maximum water and blood absorption capacity of the ACS and MACS-1/2/3. (G, H) Water and blood absorption rate of the ACS and MACS-1/2/3 within 2s. (I) Fluid simulation images of water absorption behaviors of the ACS and MACS-1/2/3. (J) Total fluid speed of the ACS and MACS-1/2/3. $n=3$, Data are means \pm SD. ns indicated no significant difference, $* P<0.05$, $\star * P<0.01, * * * P<0.001$.

\section{Shape-memory property of the MACSs}

We further evaluated the water- and blood-triggered shape-memory property of the MACSs and ACS. All sponges could be compressed and shape-fixed after squeezing out the free water (Fig. 4A, B). Upon absorbing the water, they could recover to their original shapes (Fig. 4A, C), giving a $100 \%$ recovery ratio. The recovery time $(3.3 \pm 0.6 \mathrm{~s}, 2.0 \pm 0.1 \mathrm{~s}, 1.7 \pm 0.6 \mathrm{~s})$ of the MACSs was significantly shorter than the $41 \pm$ 3.6s of the ACS (Fig. 4D and Supplementary Movies 5, 6). After absorbing blood, the shape-fixed MACSs could achieve full shape recovery ( $4.0 \pm 1.0 \mathrm{~s}, 2.5 \pm 0.5 \mathrm{~s}, 2.0 \pm 0.1 \mathrm{~s}$ ) (Supplementary Movie 7); however, the ACS kept a compressed shape and could not recover any further (Fig. 4B, D, F and Supplementary Movie 8).

The microstructure of the compressed sponges after absorbing water and blood was further observed by SEM (Fig. 4G). In their original state, homogeneous and circular microchannels with gradient numbers distributed throughout the MACSs. The circle microchannels changed to flat channels under compression stress. After absorbing water/blood, the deformed microchannels recovered to their original shapes, and the size of the microchannels had no obvious change before and after absorbing water and blood 
(Supplementary Fig. 4A-C). Furthermore, a large number of RBCs aggregated on the surface of the microchannels. The deformed micropores of the ACS recovered to their original state after absorbing water; however, they did not recover to their original shape after absorbing blood, and almost no RBCs were observed within the ACS (Fig. 4G). In addition, the shape-recovery time of the MACSs was significantly shorter (especially absorbing blood) than that of reported shape-memory hemostats (Fig. $4 \mathrm{H})$. Indeed, a large number of studies have demonstrated that, compared to water, blood is more likely to prolong the shape recovery time of hemostats due to its higher viscosity ${ }^{14,15}$. In contrast, there was no significant difference in shape recovery time for the MACSs after the absorption of water and blood. This was attributed to the highly interconnected microchannel structure, which allowed the blood to freely penetrate into the sponges. The pore structure inside the ACS and reported shape-memory hemostats generated by the removal of ice crystals and by gas foaming methods exhibited low interconnectivity, which slowed down the flow speed of the blood. $6,9,10,11,15,16$.

Fig. 4 The shape-memory property of the ACS and MACSs after absorbing water and blood. (A, B) Photographs of the water- and blood-triggered shape recovery of the ACS and MACS-1/2/3. (C, D, E, F) The shape-recovery ratio and time of the compressed sponges. (G) SEM images showing the microstructure of the compressed sponges before and after absorbing water and blood. Red arrows represented the flat channels. $(H)$ Comparison of shape-recovery time between the MACS-2/3 and reported shape-memory hemostats. $n=3$, Data are means \pm SD. ns indicated no significant difference, $\star P<0.05, * * P<0.01, * * * P<0.001$.

\section{In vitro pro-coagulant ability of the MACSs}

We also assessed the pro-coagulant ability of the gauze, GS, CELOX, CELOX-G, ACS, MCS-2, and MACSs by the $\mathrm{BCl}$ test, in which the lower the $\mathrm{BCl}$ value, the stronger the pro-coagulant ability. The $\mathrm{BCl}$ values of the MACSs decreased as the porosity increased at 5 and 10min (Fig. 5A), indicating a positive correlation between the promotion coagulation ability and porosity. The $\mathrm{BCl}$ values of the MACSs were significantly lower than that of the ACS (Fig. 5A). Also, the alkylated CS sponge (MACS-2) exhibited stronger procoagulant ability than the unmodified CS sponge (MCS-2) due to the introduction of alkyl chains $24,25,26$. Notably, the MACSs demonstrated better pro-coagulant performance compared with clinically used gauze, GS, CELOX, and CELOX-G due to the synergistic effects of the microchannel structure, CS itself, and hydrophobic modification.

The active coagulation cascade mainly relied on the aggregation of RBCs and adhesion and activation of platelets ${ }^{5}$. Thus, we further evaluated the blood coagulation effect of various samples using RBCs and platelets adhesion assays. The number of adhered RBCs and platelets to the MACSs was remarkably higher than that on the gauze, GS, CELOX, CELOX-G, ACS, and MCS-2 (Fig. 5B, C). Additionally, the higher porosity resulted in a higher number of adhered RBCs and platelets. Consistently, as observed in SEM images, more RBCs and platelets adhered to the MACSs than on other samples (Fig. 5D, E). A higher number of aggregated RBCs and activated platelets were detected in the MACSs than that in other samples (Fig. 5D, E), which accelerated blood coagulation ${ }^{31}$. CS has been proven to accelerate platelet 
adhesion and activation, and the aggregation of RBCs through electrostatic interactions ${ }^{32,33}$. The microchannel structure was able to promote penetration of the blood and aggregation of RBCs and platelets. The hydrophobic alkyl chains could insert into membranes of the RBCs and platelets, further

promoting active capture and aggregation $24,25,34$. We concluded that the CS, microchannel structure, and hydrophobic alkyl chains synergistically contributed to the strong pro-coagulant ability of the MACSs (Fig. 5F).

Fig. 5 The pro-coagulant ability of the gauze, GS, CELOX, CELOX-G, ACS, MCS-2, and MACSs. (A) The BCItime curves of various samples. $(B, C)$ The number of adhered RBCs and platelets on various samples. (D, E) SEM images showing adhesion of RBCs and platelets on various samples. (F) Schematic diagram illustrating the pro-coagulant mechanism of the MACSs. $n=3$, Data are means \pm SD. ns indicated no significant difference, ${ }^{*} P<0.01, * * P<0.01, * * * P<0.001$.

\section{In vivo hemostatic effect of the MACSs}

The MACS-2 was selected and used for in vivo hemostasis based on its mechanical strength, water/blood absorbability, blood-triggered shape-memory property, and pro-coagulant capacity (Supplementary Fig. 5). The hemostatic effect was explored in the normal rat liver perforation wound model, as illustrated in Fig. 6A. After treating the wound with the MACS-2, a small area of bloodstain was observed on the surface of the filter paper beneath the liver, while a large area of bloodstain was sighted in the gauze, GS, CELOX-G, CELOX, ACS, and MCS-2 groups (Fig. 6B and Supplementary Movie 9). Quantitatively, the total blood loss of the MACS-2 group was significantly lower than that of other groups (Fig. 6C). Also, the hemostatic time was significantly shorter than that of other groups (Fig. 6D).

Fig. 6 Hemostasis in the normal rat liver perforation wound model. (A) Schematic illustration of the hemostatic process of hemostats in a rat liver perforation wound model. (B) Photographs of the hemostatic effect of the gauze, GS, CELOX-G, CELOX, ACS, MCS-2, and MACS-2. The yellow arrow represents the bleeding site. The yellow dotted line represents the boundary of the liver. (C, D) Total blood loss and hemostatic time in the gauze, GS, CELOX-G, CELOX, ACS, MCS-2, and MACS- 2 groups. $n=3$, Data are means \pm SD. ns indicated no significant difference, ${ }^{*} P<0.01, * * P<0.01, * * * P<0.001$.

Hemorrhage control of anti-coagulated patients remains a challenge in the clinical setting ${ }^{35}$. To simulate clinical application, a heparinized-rat liver perforation wound model was used to evaluate the hemostatic capacity of various samples (Supplementary Fig. 6A). After applying the MACS-2, only a small area of bloodstain distributed on the surface of the filter paper under the liver (Supplementary Fig. 6B and Supplementary Movie 10). In contrast, a large area of bloodstain was observed after applying other hemostats. Statistical analysis showed that the hemostatic time of the MACS-2 group was much shorter than that of other groups (Supplementary Fig. $6 \mathrm{C}$ ). Also, the MACS-2 was superior in reducing the total blood loss when compared with the other hemostats (Supplementary Fig. 6D).

To further explore the clinical translation potential of the MACSs, a lethal pig liver perforation wound model was used to evaluate its hemostatic capacity (Fig. 7A). Commercial CELOX as a control is a 
commonly used hemostat in prehospital and hospital scenarios in military and civilian settings ${ }^{1,36}$. As the shape-fixed MACS-2 was filled into the wound cavity (diameter of $1.5 \mathrm{~cm}$ ), it rapidly recovered its initial cyclical shape by absorbing blood, and then filled the cavity and exerted pressure on the wound wall, achieving hemostasis within $2.0 \pm 0.5 \mathrm{~min}$ (Fig. 7B, C and Supplementary Movie 11). However, the untreated wound continued to bleed at least 10min (Supplementary Movie 12), and the CELOX-treated wound stopped bleeding at $9.0 \pm 0.3 \mathrm{~min}$ (Supplementary Movie 13). The MACS-2 was fixed on the bleeding cavity by its shape recovery. In contrast, the CELOX was prone to being washed away by the blood without external compression. In fact, manual pressing is very inconvenient in emergencies and it is difficult for the wounded to complete self-rescue on the battlefield ${ }^{31}$. We further quantified the total blood loss by determining the sum of the weight of the blood absorbed by the filter paper and hemostat. The total blood loss $(17.6 \pm 4.5 \mathrm{~g})$ in the MACS-2 group was much lower than that in untreated $(153.0 \pm$ $15.5 \mathrm{~g})$ and CELOX $(143.0 \pm 6.6 \mathrm{~g})$ groups (Fig. 7D). The MACS-2 demonstrated superior in vivo hemostatic ability for lethal noncompressible hemorrhage compared to clinically used gauze, GS, CELOX, and CELOX$\mathrm{G}$, which was due to the synergistic effect of CS itself, microchannel structure, and hydrophobic modification (Fig. 7E). The highly interconnected and controllable microchannel structure enhanced the blood adsorption capacity of the sponge, allowed the blood to perfuse into the interior of the sponge quickly, and then facilitated the recovery of its original shape, which pressed the wound and achieved rapid hemostasis. CS and alkyl chains actively captured RBCs and platelets via electrostatic and hydrophobic interactions, and also promoted aggregation of the RBCs and platelet activation. This action triggered the coagulation cascade reaction by fibrinogen-mediated interaction with the activated platelet integrin glycoprotein $\mathrm{Ilb} / \mathrm{Illa}$, which further improved hemostasis efficiency ${ }^{1,9,23}$. For clinic application, the MACSs could be customized into different shapes to meet special requirements in practical applications (Supplementary Fig. 7).

Fig. 7 Hemostasis in a lethal pig liver perforation wound model. (A) Schematic illustration of the hemostatic process of hemostats. (B) Photographs of the hemostatic effect of the blank, CELOX, and MACS-2 groups. The yellow dotted line represents the boundary of the liver. (C, D) Hemostatic time and total blood loss in the blank, CELOX, and MACS-2 groups. (E) Schematic diagram of hemostatic procedure and mechanism of the MACS-2. $n=3$, Data are means \pm SD. ns indicated no significant difference, ${ }^{\star} P<0.01, * \star P<0.01, * \star \star P<0.001$.

\section{Comparison of in vitro anti-infective property of the MACS-2 with other hemostats}

Severe bacterial infection, similar to massive blood loss, is also responsible for trauma-associated deaths ${ }^{37}$. Thus, ideal hemostats should possess robust anti-infection property. The anti-infective capacity of the MACS-2 against $S$. aureus and E. coli was evaluated by a contact-killing assay and compared with the gauze, GS, CELOX-G, CELOX, ACS, and MCS-2 (Fig. 8). Qualitative and quantitative analysis showed that, after contacting the MACS-2, the CFUs number of $S$. aureus was significantly lower than that of the gauze, GS, and ACS groups. There was no obvious difference in the CFUs number between the MACS-2 and CELOX-G, CELOX, as well as MCS-2 (Fig. 8A, C), because the hydrophobic alkyl chains could not 
interact with the membranes of $S$. aureus ${ }^{38}$. After contacting the MACS-2, the CFUs number of $E$. coli was remarkably lower than that of the gauze, GS, CELOX-G, CELOX, ACS, and MCS-2 (Fig. 8B, D). This enhanced anti-infective activity was ascribed to the synergistic effects of the microchannel structure, grafted hydrophobic alkyl chains, and CS itself 24,26 .

Fig. 8 In vitro anti-infective property of the MACS-2 and other hemostats. (A, B) Photographs of CFUs of $S$. aureus and E. coli grown on LB agar plates after contacting with TCP, gauze, GS, CELOX-G, CELOX, ACS, MCS-2, and MACS-2, respectively. (C, D) Corresponding statistical results of the CFUs of $S$. aureus and $E$. coli. $n=3$, Data are means \pm SD. ns indicated no significant difference, ${ }^{*} P<0.05,{ }^{*} * P<0.01,{ }^{*} * * P<0.001$.

\section{MACS-2 guided in situ liver regeneration}

The removal of hemostats may result in secondary bleeding and cause great distress to patients. If hemostats could be left in the injury site and directly guide in situ tissue regeneration, this would be favorable to patients and surgeons ${ }^{9}$. In situ liver regeneration as a representative model was used to evaluate the pro-regenerative ability of the MACS-2 and ACS. Rapid host cell infiltration was the first and crucial step for endogenous tissue regeneration ${ }^{39,40}$. DAPI and H\&E staining showed that the host cells migrated into the interior of the MACS-2, but were mainly distributed around the edge of the ACS due to its dense structure (Fig. 9A). Accordingly, the cell number inside the MACS-2 was significantly higher than that of the ACS (Fig. 9B). Infiltrated cells secreted a large amount of extracellular matrix and formed neotissue. The tissue ingrowth area within the MACS-2 was much larger than that of the ACS. However, almost no neotissue grew inside the ACS (Fig. 9A, C). A rich capillary network capable of delivering adequate oxygen and nutrients is indispensable for newly formed tissue survival. Thus, vascularization was assessed by immunostaining for von Willebrand Factor (VWF). A high density of capillaries distributed inside the MACS-2 (Fig. 9D); in contrast, almost no capillary was observed within the ACS. A large number of ALB positive cells were observed in the interior of the MACS-2, indicating ingrowth of liver parenchymal cells and liver tissue regeneration. In comparison, almost no liver parenchymal cells infiltrated into the ACS (Fig. 9A, E) ${ }^{41}$. The improved ability of cellularization, vascularization, and tissue ingrowth of the MACS-2 attributed to the highly interconnected microchannels, high porosity, and good biocompatibility (Fig. 9F) ${ }^{39}$. To our knowledge, there has not been any report to date regarding the use of a shape-memory hemostatic sponge for internal penetrating wound repair. Our MACS-2 simultaneously achieved hemostasis and in situ tissue regeneration, which broadens the application of hemostats and opens up an opportunity for the design and construction of clinically beneficial hemostats. Specifically, the application of our hemostatic sponge will reduce patient discomfort, simplify treatment procedures, and potentially decrease healthcare costs.

Fig. 9 Liver regeneration in rat models after implantation of the ACS and MACS-2. (A) DAPI staining showing cell infiltration within the ACS and MACS-2. H\&E staining showing tissue ingrowth. Yellow asterisk $\left(^{*}\right)$ represents the alkylated CS. Images of immunofluorescent staining for VWF (red) and ALB (red) indicating capillary and liver parenchymal cell (LPC) infiltration within the ACS and MACS-2. Yellow pound key (\#) and arrow represent capillary and LPC, respectively. (B, C, D, E) Quantification of cell 
number, tissue ingrowth area, capillary number, and LPCs per view within the ACS and MACS-2. (F) Schematic illustration of in situ liver regeneration, including the host cell infiltration and vascularization. $n=3$, Data are means \pm SD. ns indicated no significant difference, ${ }^{*} P<0.05, * * P<0.01, * * * P<0.001$.

\section{Conclusion}

In this study, we incorporated a microchannel structure into a CS sponge and further modified it with hydrophobic alkyl chains. The MACSs achieved rapid shape recovery by absorption of water and blood. Compared with clinically used gauze, GS, CELOX, and CELOX-G, the MACSs demonstrated stronger procoagulant ability in vitro and hemostatic capacity in lethally normal/heparinized rat and normal pig liver perforation wound models. Moreover, they exhibited enhanced anti-infective activity against $S$. aureus and E. coli. Notably, the MACSs could be left in the wound bed and guided in situ liver regeneration. All results in this study indicate that MACSs have the clinical translational capacity to provide effective treatment of potentially lethal noncompressible hemorrhage and to facilitate tissue repair.

\section{Materials And Methods}

\section{Materials}

Chitosan (CS, molecular mass of 100 kDa) was from Jinan Haidebei Biotech Co., Ltd., China. Dodecyl aldehyde (DA, 99.5\%) and sodium cyanoborohydride (NaCNBH3, 95\%) were from Shanghai Aladin Co., Ltd., China. Polylactic acid (PLA) filament was from Jinluotuo Biotech Co., Ltd., China. Acetic acid, dichloromethane, and ethyl alcohol were from Tianjin Reagent Co., Ltd., China. All chemicals were of analytical grade.

\section{Fabrication of the MACSs}

The fabrication of the MACSs was as follows: First, the PLA microfiber templates with filling ratios of 20, 40, and 60\% were printed using a 3D printer (Shenzhen Creality 3D Tech Co., LTD., China). Second, the templates were filled with CS solution (1, 2, and 4\%, w/v) dissolved in acetic acid aqueous solution (2\%, $\mathrm{v} / \mathrm{v})$, followed by freezing in liquid nitrogen and lyophilization. Third, the CS sponges with microchannel structure were obtained by leaching out the templates with dichloromethane. Residual acetic acid was neutralized with a mixed solution of ethyl alcohol/ $\mathrm{NaOH}(9 / 1, \mathrm{v} / \mathrm{v})$. The resultant CS sponges were further modified with DA in the presence of $\mathrm{NaCNBH}$. Unreacted DA and NaCNBH3 were removed by rinsing with ethyl alcohol and deionized water (DIW) in turn. The MACSs generated from PLA microfiber templates with filling ratios of 20,40 , and $60 \%$ were named as the MACS-1, MACS-2, and MACS-3, respectively. An unmodified microchannelled CS sponge generated from a PLA microfiber template with a ratio of $40 \%$ was abbreviated as the MCS-2. The alkylated CS sponge prepared by direct freeze-drying was named ACS.

\section{FTIR spectrum test}


The spectra of CS powder, PLA microfiber template, PLA/CS composite, and microchannelled CS sponge were recorded in the range of $4000-500 \mathrm{~cm}-1$ by using a fourier transform infrared spectrometer (FTIR, TENSOR II, Germany).

\section{XPS analysis}

The superficial chemical structure and element content of the CS sponges with or without modification were detected using an X-ray photoelectron spectrometer (XPS, Axis Ultra DLD, England).

\section{Characterization of macro/microstructure and porosity}

The macro and microstructure of the MACSs and ACS were characterized by the micro-computed tomography (Micro-CT, Germany) and scanning electron microscopy (SEM, MERLIN Compact, Germany) ${ }^{19}$. The average pore size was measured using Image-J software (ImageJ 1.44p). The porosity was calculated using Micro-CT.

\section{Mechanical test}

The mechanical strength of the MACSs generated with different CS concentrations $(1,2$, and $4 \%, w / v)$ and PLA microfiber filling ratios $(20,40$, and $60 \%$ ) were prepared into cylindrical shapes ( $5 \mathrm{~mm}$ in height and $8 \mathrm{~mm}$ in diameter) and tested in a universal mechanical tester (Instron, 3345). The compression strain and speed were fixed at $70 \%$ and $1 \mathrm{~mm} / \mathrm{min}$, respectively. The maximum compressive stress was obtained from a stress-strain curve. The compressive stress of the MACSs $(5 \mathrm{~mm}$ in height and $8 \mathrm{~mm}$ in diameter) after absorbing the blood was also measured.

\section{Water/blood absorption behavior}

After squeezing out water, the compressed MACSs and ACS contacted the water and blood. Their positions in water and blood were recorded by a digital camera. To quantitatively evaluate absorption behavior, the compressed MACSs and ACS were weighed $\left(W_{d}\right)$ and soaked into water and blood from rats. At different time intervals, they were taken out and weighted $\left(W_{w}\right)$. The water/blood absorption capacity was calculated according to the following equation:

Water/blood absorption capacity $(\mathrm{g} / \mathrm{g})=\left(\mathrm{W}_{\mathrm{w}} \otimes \mathrm{W}_{\mathrm{d}}\right) / \mathrm{W}_{\mathrm{d}}$

The water/blood absorption rate was calculated by measuring the slope of the water/blood absorption capacity-time curve within 2s. Moreover, the absorption behavior was further measured by digital fluid simulation. The MACSs and ACS were modeled by using the software Solidworks Flow Simulation. The flow orientation of water with a dynamic viscosity of $1.7912 \times 10-3 \mathrm{~Pa}$. $\mathrm{s}$ was parallel to the axial direction of the sponges. The working temperature and pressure were set as $273.2 \mathrm{~K}$ and $101325 \mathrm{~Pa}$, respectively. The mass flow at the inlet was $0.001 \mathrm{~m} / \mathrm{s}$. The stimulated pore size was consistent with statistical results from SEM images. To simplify the simulation, the micropore with low interconnectivity in the sponge was replaced by microchannel with comparable size to the micropore. 


\section{Shape-memory property}

We assessed the shape-memory property of the MACSs and ACS using the reported method ${ }^{6}$. The MACSs and ACS were first compressed to squeeze out the free water. Then, both water and blood were dropped onto their top surfaces. The shape-recovery process was recorded using a digital camera. The shaperecovery ratio and time were measured. Also, the microstructure recovery of the MACSs and ACS before and after absorbing water and blood was further observed by SEM.

\section{Blood clotting index test}

The pro-coagulant ability of the MACSs was evaluated by measuring the blood clotting index $(\mathrm{BCl})^{27,28}$. The gauze, gelatin sponge (GS), CELOX, CELOX-gauze (CELOX-G), ACS, and MCS-2 were used as controls. The MACSs were compressed to squeeze out water and placed in EP tubes. After warming for $10 \mathrm{~min}$ at $37^{\circ} \mathrm{C}, 50 \mu \mathrm{L}$ of the citrated whole blood (CWB) from rats was dropped onto their top surfaces. After incubation for 5 and $10 \mathrm{~min}$ at $37^{\circ} \mathrm{C}, 3 \mathrm{~mL}$ of DIW was added into each EP tube, and optical density (OD) value at $540 \mathrm{~nm}$ of the supernatant was determined using a microplate reader (BIO-RAD, iMARKTM). The mixed DIW/CWB $(3 \mathrm{~mL} / 50 \mu \mathrm{L})$ solution was used as a negative control and its $\mathrm{OD}_{540 \mathrm{~nm}}$ value represented as $100 \%$. The $\mathrm{BCl}$ was calculated based on the following equation:

$\mathrm{BCl}(\%)=O \mathrm{D}_{\text {material }} / \mathrm{OD}_{\text {reference }}$ value $\times 100 \%$

\section{RBCs and platelets adhesion assays}

The interactions between the MACSs and RBCs were investigated with the previously reported method with some modification ${ }^{27}$. The gauze, GS, CELOX, CELOX-G, ACS, and MCS-2 were used as controls. Before testing, RBCs suspension was obtained by centrifuging the CWB for $10 \mathrm{~min}$ under $400 \mathrm{G}$. The MACSs were compressed to drain off water and placed in a 24-well microplate. Next, $100 \mu \mathrm{L}$ of RBCs suspension was dropped onto their top surfaces. After incubation for $30 \mathrm{~min}$ at $37^{\circ} \mathrm{C}$, they were rinsed with a phosphate buffer solution (PBS, $\mathrm{pH}=7.4$ ) to remove nonadherent $\mathrm{RBC}$, and then transferred into DIW ( $4 \mathrm{~mL})$ to lyse adhered RBCs to release hemoglobin. After $1 \mathrm{~h}, 100 \mu \mathrm{L}$ of the supernatant was taken out and placed into a 96-well microplate followed by measuring its $\mathrm{OD}_{540 \mathrm{~nm}}$ value. The $\mathrm{OD}_{540 \mathrm{~nm}}$ value of a solution composed of $100 \mu \mathrm{L}$ of RBCs suspension and $4 \mathrm{~mL}$ of DIW was used as a reference value. The percentage of adhered RBCs was calculated by the following equation:

RBCs adhesion $(\%)=O D_{\text {material }} / O D_{\text {reference }}$ value $\times 100 \%$

The interactions between various hemostats and platelets were further evaluated by a platelet adhesion assay ${ }^{27}$. Before measurement, the platelet-rich plasma (PRP) was obtained by centrifuging the CWB for 10 min under 400G. The MACSs were compressed to squeeze out water and placed into a 24-well microplate. Then, $100 \mu \mathrm{L}$ of PRP was dropped on their top surfaces followed by incubation for $30 \mathrm{~min}$ at $37^{\circ} \mathrm{C}$. Next, they were washed with PBS to remove nonadherent platelets and soaked into a $1 \%$ Triton X100 solution to lyse platelets to release the lactate dehydrogenase (LDH) enzyme. The LDH was 
determined with an LDH kit (Biyuntian, China) according to its instruction. Finally, the $\mathrm{OD}_{490 \mathrm{~nm}}$ value of the supernatant was measured. The $\mathrm{OD}_{490 \mathrm{~nm}}$ value of a solution composed of $100 \mu \mathrm{L}$ of PRP unexposed with hemostats was measured and used as a reference value. The percentage of adhered platelets was calculated by the following equation:

Adhered platelets $(\%)=O D_{\text {material }} / O D_{\text {reference }}$ value $\times 100 \%$

The adherence of RBCs and platelets on the various hemostats was observed by SEM. Briefly, hemostats were placed into each well in a 24-well microplate and contacted with $100 \mu \mathrm{L}$ of RBCs and PRP suspensions. After $30 \mathrm{~min}$ at $37^{\circ} \mathrm{C}$, they were rinsed with PBS, and then fixed with $2.5 \%$ glutaraldehyde and dehydrated using a series of graded alcohol solutions. After drying, they were cut, and the longitudinal sections were sputtered with gold and observed by SEM.

\section{Hemostasis in vivo}

The hemostatic ability of the MACS-2 was evaluated by lethally normal/heparinized rat and normal pig liver perforation wound models, and compared with gauze, GS, CELOX, CELOX-G, ACS, and MCS-2. All animal experiments were performed with the approval of the Animal Experimental Ethics Committee of Nankai University.

Normal and heparinized rat liver perforation wound models: A rat (male, weight of 250 300 g) was anesthetized by injecting $10 \mathrm{wt} \%$ chloral hydrate in a dose of $1 \mathrm{~mL} / 300 \mathrm{~g}$. Then, the rat's abdomen was incised, and the liver was lifted and placed onto the surface of the pre-weighted filter paper. Next, a circular perforation wound (diameter of $6 \mathrm{~mm}$ ) was created on the liver to induce hemorrhaging. Finally, the cylindrical MACS-2 (diameter of $8 \mathrm{~mm}$ ) was compressed to squeeze out water and filled into the wound cavity. The hemostatic process was recorded with a digital camera. The blood loss was measured by determining the total weight of the blood absorbed by the filter paper and hemostats. The hemostatic time was recorded with a timer. The heparin solution (50UI) was injected into the rat (male, weight of $250 \sim 300 \mathrm{~g}$ ) through a vein at a dose of $2 \mathrm{~mL} / \mathrm{kg}$ and used for the construction of the heparinized rat liver perforation wound model. Other procedures were similar to the method mentioned above.

Lethal pig liver perforation wound model: Bama miniature pig ( 3 months, weight of $15 \mathrm{~kg}$ ) was anesthetized by injecting a mixed solution of midazolam and xylazine hydrochloride $(2 / 1, \mathrm{v} / \mathrm{v})$ into its muscle at a dose of $0.14 \mathrm{~mL} / 1 \mathrm{~kg}$. Then, the abdomen of the pig was incised, and its liver was taken out and placed onto the surface of the filter paper. Next, a $15 \mathrm{~mm}$-diameter circular perforation wound was made on the liver. After bleeding, the cylindrical MACS-2 (diameter of $18 \mathrm{~mm}$ ) was compressed to squeeze out the free water and filled into the wound cavity. The hemostatic process was recorded with a digital camera. The total blood loss from each liver was weighed and the hemostatic time were recorded.

\section{In situ liver regeneration}


The in situ pro-regenerative ability of the MACS-2 and ACS was evaluated using a representative rat liver defect model. A rat (male, weight of $200 \sim 300 \mathrm{~g}$ ) was anesthetized with $10 \mathrm{wt} \%$ chloral hydrate, and its abdomen was incised. Then, a $6 \mathrm{~mm}$-diameter circular perforation wound was created on the liver. Next, the cylindrical MACS-2 was compressed and filled into the wound. As a comparison, uncompressed ACS was also filled into the wound. After hemostasis, the abdomen was sutured, and the rat was feed normally. After one-month post-surgery, the rat was paralyzed, and the liver was taken out for histological and immunofluorescence staining. H\&E staining was used to assess tissue ingrowth. DAPI staining was used to evaluate the host cell infiltration. Immunofluorescence staining for von Willebrand factor (vWF) and albumin (ALB) was performed to evaluate vascularization and liver parenchymal cell infiltration.

\section{In vitro anti-infective activity}

The in vitro anti-infective activity of the MACS-2 against $S$. aureus (ATCC6538) and E. coli (ATCC25922) was tested by a contact-killing assay ${ }^{24}$. Tissue culture plate (TCP), gauze, GS, CELOX, CELOX-G, ACS, and MCS-2 were used as controls. Before the test, the MACS-2 was compressed to squeeze out water and placed into each well in a 48-well microplate. After sterilization for $1 \mathrm{~h}$ under UV irradiation, $10 \mu \mathrm{L}$ of the bacterial suspension with a concentration of $10^{8}$ colony forming units $/ \mathrm{milliliter}(\mathrm{CFUs} / \mathrm{mL}$ ) was dropped onto their top surface. After $2 \mathrm{~h}$ at $37^{\circ} \mathrm{C}$, the survival bacteria were resuspended by adding $200 \mu \mathrm{L}$ of sterilized PBS into each well. Next, $20 \mu \mathrm{L}$ of resuspended bacterial suspension was taken out and diluted five times by ten-fold dilution to obtain a final diluting bacterial suspension (FDBS). Subsequently, 20 $\mathrm{L}$ of FDBS was spread onto the surface of the LB agar plate and incubated at $37^{\circ} \mathrm{C}$. After incubation overnight, the formed CFUs on each LB agar plate were counted.

\section{Statistical analysis}

All tests were processed in triplicate. Each group has at least three parallel samples. Statistical analyses were performed using GraphPad Prism 5 software. Values are expressed as the means \pm standard deviation (SD). The One-way ANOVA with Newman-keuls multiple comparison test was used to evaluate the statistical differences between groups. ${ }^{\star} P<0.05$ was considered to be statistically significant.

\section{Declarations}

\section{Data availability}

The authors declare that all data supporting the findings of this study are available within the paper and its Supplementary Material files or are available from the authors upon request.

\section{Acknowledgements}

The authors greatly thank Phillip Bryant for her help on the language revision. This work was financially supported by the National Key Research and Development Program of China (2016YFC1101304), 
National Natural Science Foundation of China (31670990) and National Natural Science Foundation of China (81972063).

\section{Author contributions}

M. L. W. and X. D. conceived the research; X. D., L. W. and H. Y. designed the experiments; X. D., L. W., H. Y., Z. J., S. L. and Z. C. performed the experiments; W. L. and Y. B. characterized the structure of the sponges; X. D., L. W., M. Z. and D. K. interpreted the data, analysed the data and wrote the manuscript. All authors discussed the data and direction of the project at regular intervals throughout the study.

\section{Competing interests}

There are no competing interests.

\section{References}

1. Hickman, D. A., Pawlowski, C. L., Sekhon, U. D. S., Marks, J. \& Gupta, A. S. Biomaterials and advanced technologies for hemostatic management of bleeding. Adv. Mater. 30, 1700859 (2018).

2. Gao, Y. et al. A polymer-based systemic hemostatic agent. Sci. Adv. 6, 0588 (2020).

3. Johnson, D. et al. The effects of QuikClot Combat Gauze on hemorrhage control in the presence of hemodilution and hypothermia. Ann. Med. Surg. 3, 21-25 (2014).

4. Boerman, M. et al. Next generation hemostatic materials based on NHS-ester functionalized poly(2oxazoline)s. Biomacromolecules 18, 2529-2538 (2017).

5. Yang, X. et al. Peptide-immobilized starch/PEG sponge with rapid shape recovery and dual-function for both uncontrolled and noncompressible hemorrhage. Acta Biomater. 99, 220-235 (2019).

6. Zhao, X., Guo, B., Wu, H., Liang, Y. \& Ma, P. X. Injectable antibacterial conductive nanocomposite cryogels with rapid shape recovery for noncompressible hemorrhage and wound healing. Nat. Commun. 9, 2784 (2018).

7. Bu, Y. et al. Synthesis and properties of hemostatic and bacteria-responsive in situ hydrogels for emergency treatment in critical situations. ACS Appl. Mater. Interfaces 8, 12674-12683 (2016).

8. Landsman, T. L. et al. A shape memory foam composite with enhanced fluid uptake and bactericidal properties as a hemostatic agent. Acta Biomater. 47, 91-99 (2017).

9. Yang, X. et al. Design and development of polysaccharide hemostatic materials and their hemostatic mechanism. Biomater. Sci. 5, 2357-2368 (2017)

10. Chen, S., Carlson, M. A., Zhang, Y. S., Hu, Y. \& Xie, J. Fabrication of injectable and superelastic nanofiber rectangle matrices ("peanuts") and their potential applications in hemostasis. Biomaterials 179, 46-59 (2018).

11. Mueller, G. R. et al. A novel sponge-based wound stasis dressing to treat lethal noncompressible hemorrhage. J. Trauma Acute Care Surg. 73, S134-S139 (2012). 
12. Rodriguez, J. N. et al. Opacification of shape memory polymer foam designed for treatment of intracranial aneurysms. Ann. Biomed. Eng. 40, 883-897 (2012).

13. Rodriguez, J. N. et al. In vivo response to an implanted shape memory polyurethane foam in a porcine aneurysm model. J. Biomed. Mater. Res. A 102, 1231-1242 (2014).

14. Huang, Y. et al. Degradable gelatin-based IPN cryogel hemostat for rapidly stopping deep noncompressible hemorrhage and simultaneously improving wound healing. Chem. Mater. 32, 65956610 (2020).

15. Li, M., Zhang, Z., Liang, Y., He, J. \& Guo, B. Multifunctional tissue-adhesive cryogel wound dressing for rapid nonpressing surface hemorrhage and wound repair. ACS Appl. Mater. Interfaces 12, 3585635872 (2020).

16. Xu, C., Dai, G. \& Hong, Y. Recent advances in high-strength and elastic hydrogels for 3D printing in biomedical applications. Acta Biomater. 95, 50-59 (2019).

17. Daly, A. C., Pitacco, P., Nulty, J., Cunniffe, G. M. \& Kelly, D. J. 3D printed microchannel networks to direct vascularisation during endochondral bone repair. Biomaterials 162, 34-46 (2018).

18. Rnjak, K. J., Wray, L. S., Golinski, J. M. \& Kaplan, D. L. Arrayed hollow channels in silk-based scaffolds provide functional outcomes for engineering critically-sized tissue constructs. Adv. Funct. Mater. 24, 2188-2196 (2014).

19. Zhu, M. et al. In vivo engineered extracellular matrix scaffolds with instructive niches for oriented tissue regeneration. Nat. Commun. 10, 4620 (2019).

20. Zhang, L., Yang, G., Johnson, B. N. \& Jia, X. Three-dimensional (3D) printed scaffold and material selection for bone repair. Acta Biomater. 84, 16-33 (2019).

21. Zhao, Y. et al. Synthetic poly(vinyl alcohol)-chitosan as a new type of highly efficient hemostatic sponge with blood-triggered swelling and high biocompatibility. J. Mater. Chem. B 7, 1855-1866 (2019).

22. Leonhardt, E. E., Kang, N., Hamad, M. A., Wooley, K. L. \& Elsabahy, M. Absorbable hemostatic hydrogels comprising composites of sacrificial templates and honeycomb-like nanofibrous mats of chitosan. Nat. Commun. 10, 2307 (2019).

23. Du, X. et al. Injectable hydrogel composed of hydrophobically modified chitosan/oxidized-dextran for wound healing. Mater. Sci. Eng. C 104, 109930 (2019).

24. Du, X. et al. Anti-infective and pro-coagulant chitosan-based hydrogel tissue adhesive for sutureless wound closure. Biomacromolecules 21, 1243-1253 (2020).

25. Dowling, M. B., Kumar, R., Keibler, M. A., Hess, J. R., Bochicchio, G, V. \& Raghavan, S. R. A selfassembling hydrophobically modified chitosan capable of reversible hemostatic action. Biomaterials 32, 3351-3357 (2011).

26. Chen, G., Yu, Y., Wu, X., Wang, G., Ren, J. \& Zhao, Y. Wound healing: Bioinspired multifunctional hybrid hydrogel promotes wound healing. Adv. Funct. Mater. 28, 1870233 (2018). 
27. Wang, C., Niu, H., Ma, X., Hong, H., Yuan, Y. \& Liu, C. Bioinspired, injectable, quaternized hydroxyethyl cellulose composite hydrogel coordinated by mesocellular silica foam for rapid, noncompressible hemostasis and wound healing. ACS Appl. Mater. Interfaces 11, 34595-34608 (2019).

28. Zhang, Z. et al. Sandwich-like fibers/sponge composite combining chemotherapy and hemostasis for efficient postoperative prevention of tumor recurrence and metastasis. Adv. Mater. 30, 1803217 (2018).

29. Fang, Y. et al. 3D porous chitin sponge with high absorbency, rapid shape recovery, and excellent antibacterial activities for noncompressible wound. Chem. Eng. J. 388, 124169 (2020).

30. Gupta, D., Singh, A, K., Dravid, A. \& Bellare, J. Multiscale porosity in compressible cryogenically 3D printed gels for bone tissue engineering. ACS Appl. Mater. Interfaces 11, 20437-20452 (2019).

31. Zhao, X., Liang, Y., Guo, B., Yin, Z., Zhu, D. \& Han, Y. Injectable dry cryogels with excellent bloodsucking expansion and blood clotting to cease hemorrhage for lethal deep-wounds, coagulopathy and tissue regeneration. Chem. Eng. J. 403, 126329 (2021).

32. Benesch, J. \& Tengvall, P. Blood protein adsorption onto chitosan. Biomaterials 23, 2561-2568 (2002).

33. Chan, L. W., Kim, C. H., Wang, X., Pun, S. H., White, N. J. \& Kim, T. H. PolySTAT-modified chitosan gauzes for improved hemostasis in external hemorrhage. Acta Biomater. 31, 178-185 (2016).

34. Cui, C. et al. Water-triggered hyperbranched polymer universal adhesives: From strong underwater adhesion to rapid sealing hemostasis. Adv. Mater. 31, 1905761 (2019).

35. Bu, Y. et al. Tetra-PEG based hydrogel sealants for in vivo visceral hemostasis. Adv. Mater. 31, 1901580 (2019).

36. Liu, C. et al. A highly efficient, in situ wet-adhesive dextran derivative sponge for rapid hemostasis. Biomaterials 205, 23-37 (2019).

37. Yang, X. et al. Fabricating antimicrobial peptide-immobilized starch sponges for hemorrhage control and antibacterial treatment. Carbohyd. Polym. 222, 115012 (2019).

38. Vo, D. \& Lee, C. K. Antimicrobial sponge prepared by hydrophobically modified chitosan for bacteria removal. Carbohyd. Polym. 187, 1-7 (2018).

39. Wu, P. et al. Construction of vascular graft with circumferentially oriented microchannels for improving artery regeneration. Biomaterials 242, 119922 (2020).

40. Li, W. et al. Subcutaneously engineered autologous extracellular matrix scaffolds with aligned microchannels for enhanced tendon regeneration: Aligned microchannel scaffolds for tendon repair. Biomaterials 224, 119488 (2019)

41. Cao, L. et al. Construction of multicellular aggregate by E-cadherin coated microparticles enhancing the hepatic specific differentiation of mesenchymal stem cells. Acta Biomater. 95, 382-394 (2019).

\section{Figures}




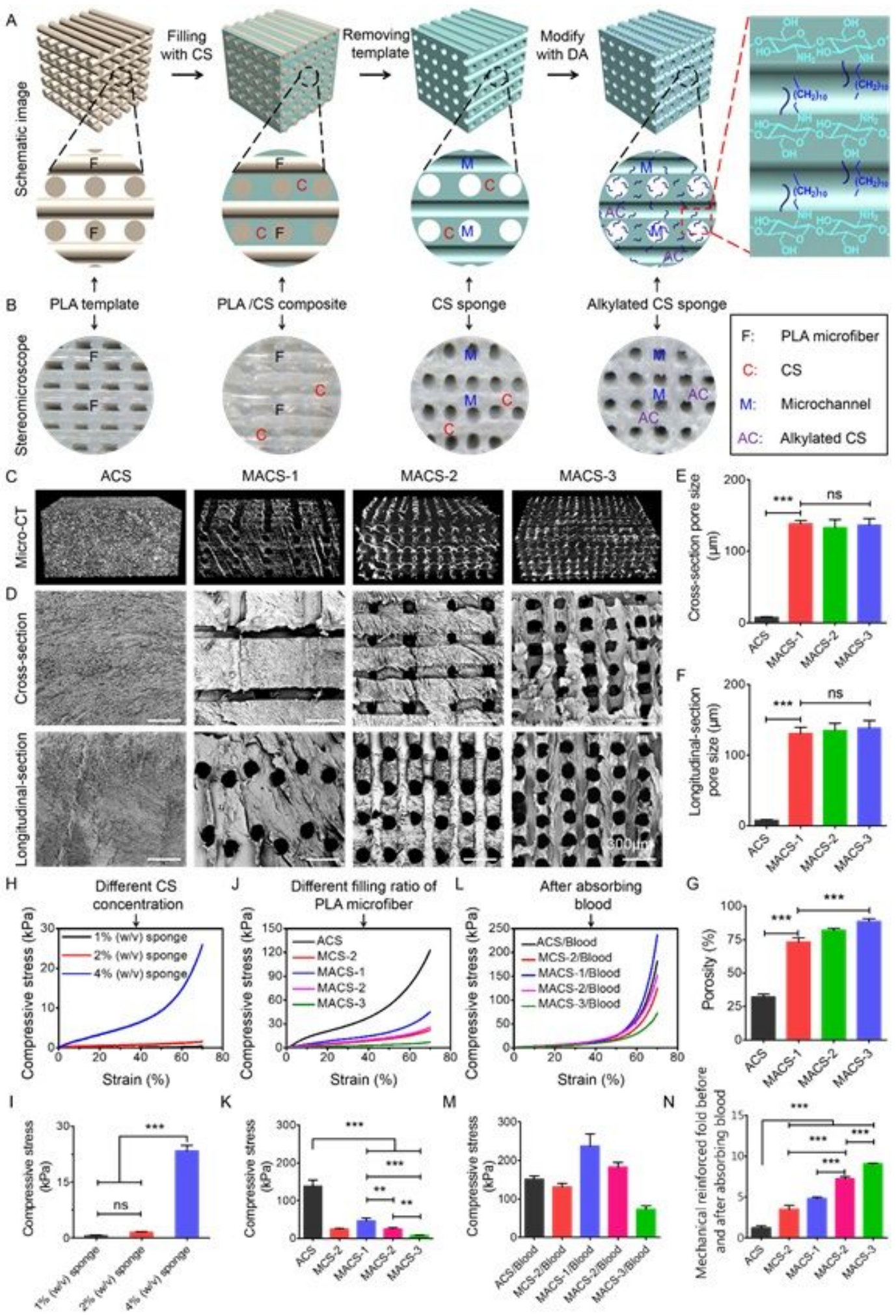

Figure 1

Fabrication and characterization of the MACSs with different porosity. (A) Schematic illustration of the fabrication process of the MACSs. (B) Stereomicroscopic images of the PLA microfiber template, CS/PLA composite, microchannelled CS sponge, and microchannelled alkylated CS sponge. (C, D) Micro-CT and SEM images showing the macro and microstructure of the ACS and MACS-1/2/3. (E, F) The pore size of the ACS and MACS-1/2/3 in cross-section and longitudinal-section. (G) The porosity of the ACS and 
MACS-1/2/3. (H, I) Compressive stress-strain curves and compressive stress of the MACSs with different CS concentrations $(1,2$, and $4 \%(w / v)) .(J, K, L, M)$ Compressive stress-strain curves and compressive stress of the ACS, MCS-2, and MACS-1/2/3 before and after absorbing blood. (N) Mechanically reinforced folds of the ACS, MCS-2, and MACS-1/2/3 before and after absorbing blood. $n=3$, Data are means \pm SD. ns indicated no significant difference, ${ }^{*} P<0.05,{ }^{*} \mathrm{P}<0.01, * \star * P<0.001$.
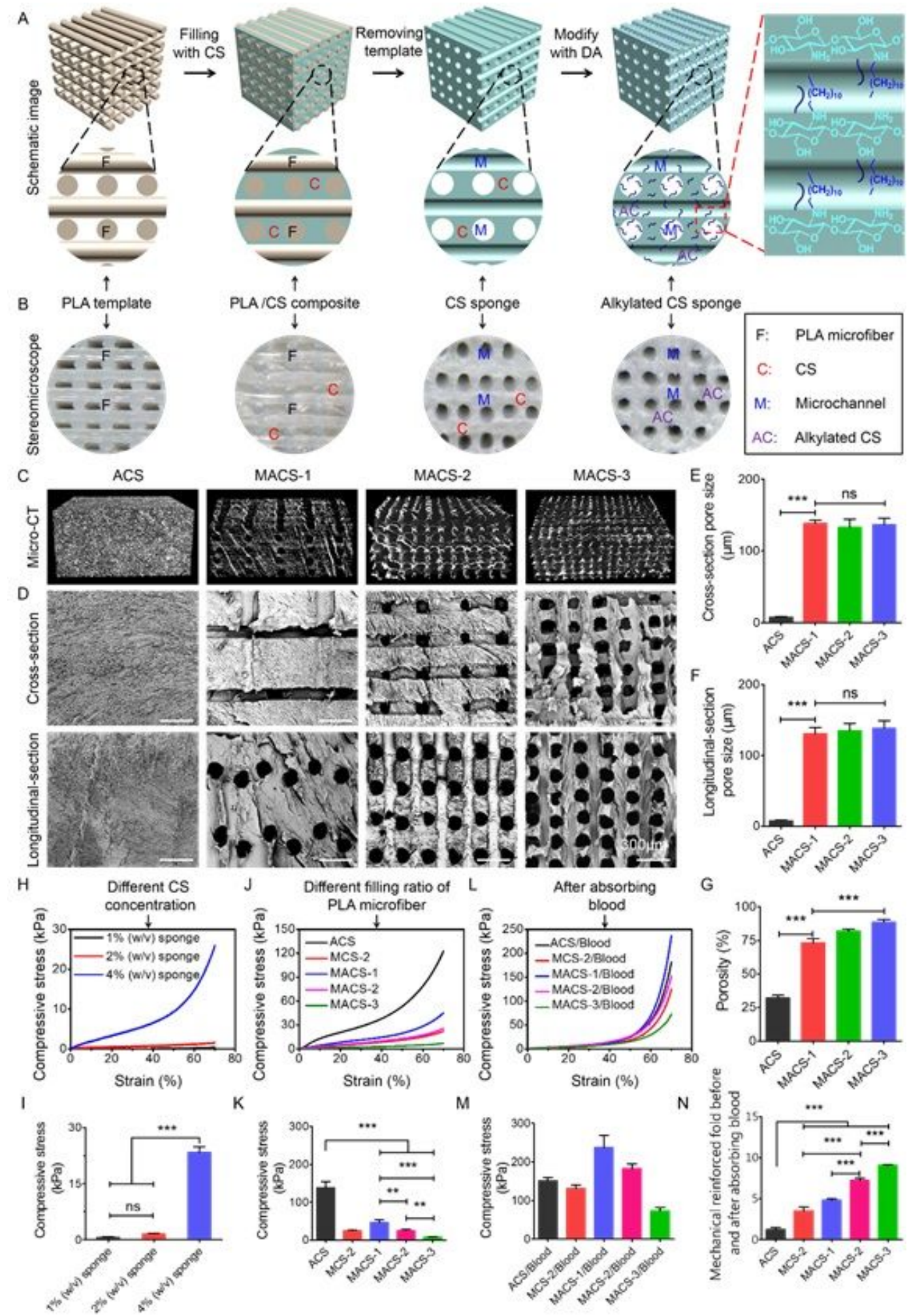

Figure 1 
Fabrication and characterization of the MACSs with different porosity. (A) Schematic illustration of the fabrication process of the MACSs. (B) Stereomicroscopic images of the PLA microfiber template, CS/PLA composite, microchannelled CS sponge, and microchannelled alkylated CS sponge. (C, D) Micro-CT and SEM images showing the macro and microstructure of the ACS and MACS-1/2/3. (E, F) The pore size of the ACS and MACS-1/2/3 in cross-section and longitudinal-section. (G) The porosity of the ACS and MACS-1/2/3. (H, I) Compressive stress-strain curves and compressive stress of the MACSs with different CS concentrations $(1,2$, and $4 \%(w / v)) .(J, K, L, M)$ Compressive stress-strain curves and compressive stress of the ACS, MCS-2, and MACS-1/2/3 before and after absorbing blood. (N) Mechanically reinforced folds of the ACS, MCS-2, and MACS- $1 / 2 / 3$ before and after absorbing blood. $n=3$, Data are means \pm SD. ns indicated no significant difference, ${ }^{*} P<0.05,{ }^{*} \mathrm{P}<0.01$, $* * * \mathrm{P}<0.001$.

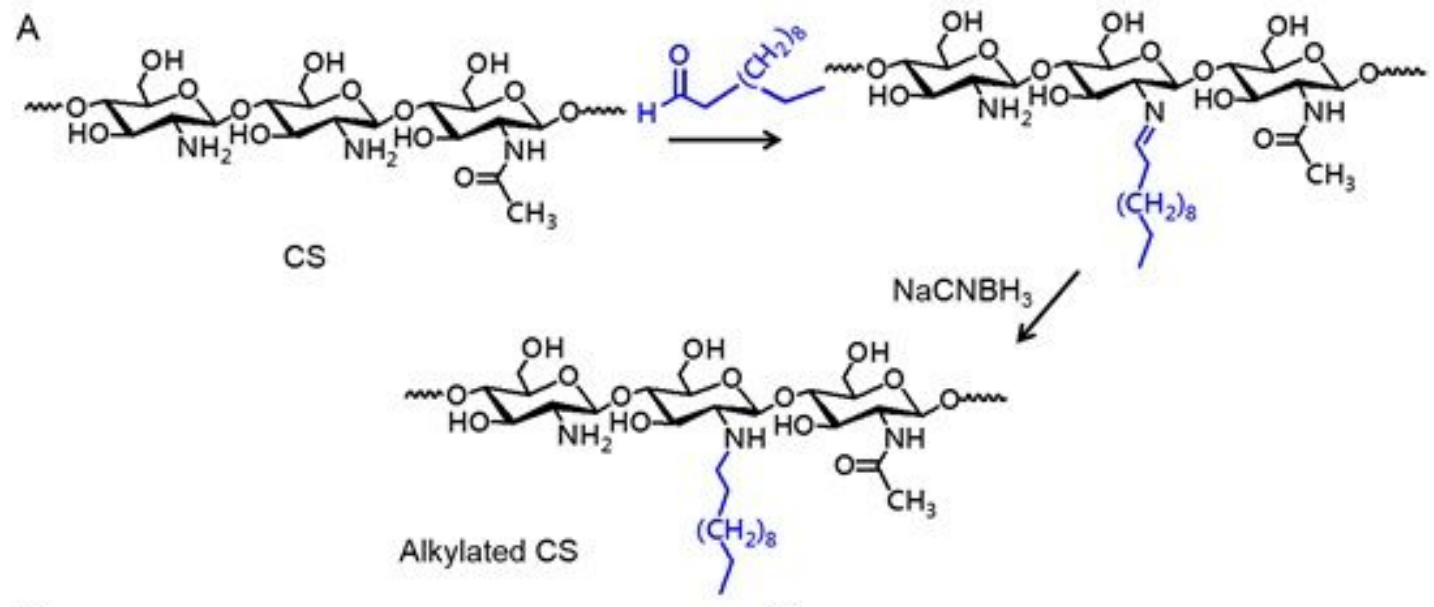

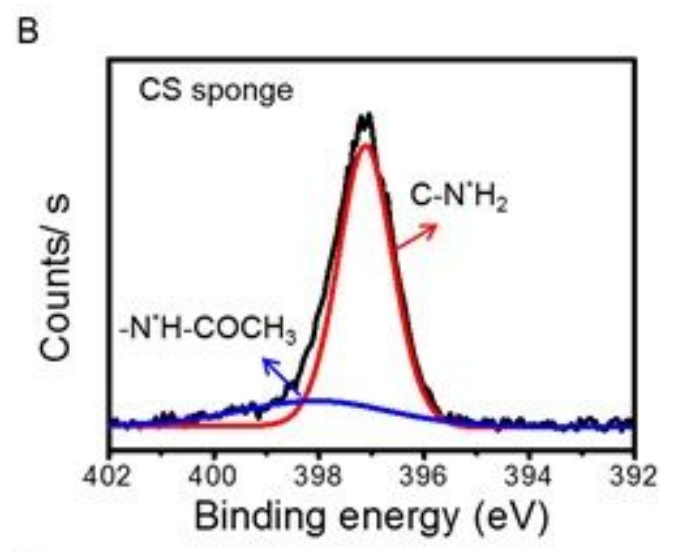

D

Table 1. The area of $\mathrm{N} 1 \mathrm{~s}$ peak

\begin{tabular}{cccc}
\hline Sponge & $\begin{array}{c}\mathrm{C}-\mathrm{N}^{\prime} \mathrm{H}_{2} \\
\%\end{array}$ & $\begin{array}{c}-\mathrm{N}^{\prime} \mathrm{H}-\mathrm{COCH}_{3} \\
\%\end{array}$ & $\begin{array}{c}\mathrm{C}-\mathrm{N} \cdot \mathrm{H}-\mathrm{C} \\
\%\end{array}$ \\
\hline $\mathrm{CS}$ & 82.08 & 17.92 & 0.00 \\
Alkylated $\mathrm{CS}$ & 51.05 & 9.11 & 39.87 \\
\hline
\end{tabular}

C

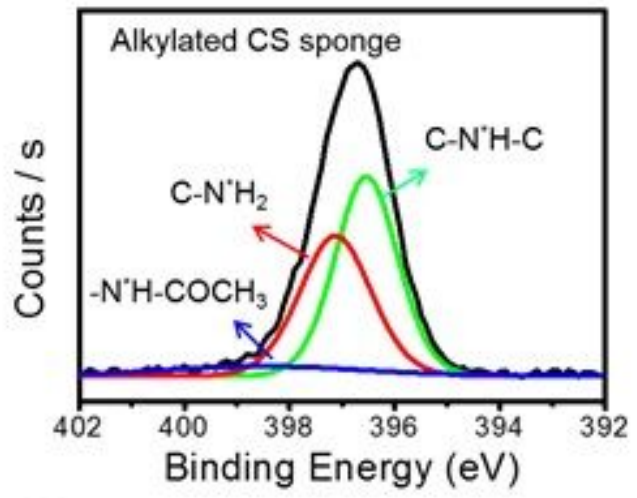

E

Table 2. The calibrate value of $\mathrm{C} 1 \mathrm{~s}$

\begin{tabular}{ccc}
\hline Sponge & $\begin{array}{c}\text { Atomic } \\
\text { Conc \% }\end{array}$ & $\begin{array}{c}\text { Mass } \\
\text { Conc \% }\end{array}$ \\
\hline CS & 46.86 & 39.86 \\
\hline Alkylated CS & 60.16 & 53.67 \\
\hline
\end{tabular}

Figure 2

Chemical characterization of the MACSs. (A) Modification of the CS sponge with DA in the presence of $\mathrm{NaCNBH} 3$ as a reducing agent. $(\mathrm{B}, \mathrm{C})$ XPS spectra showing N1s peak of the CS and alkylated CS sponges. $(D, E)$ The area of N1s peak and the calibrate value of $\mathrm{C} 1 \mathrm{~s}$ in the $\mathrm{CS}$ and alkylated CS sponges. 


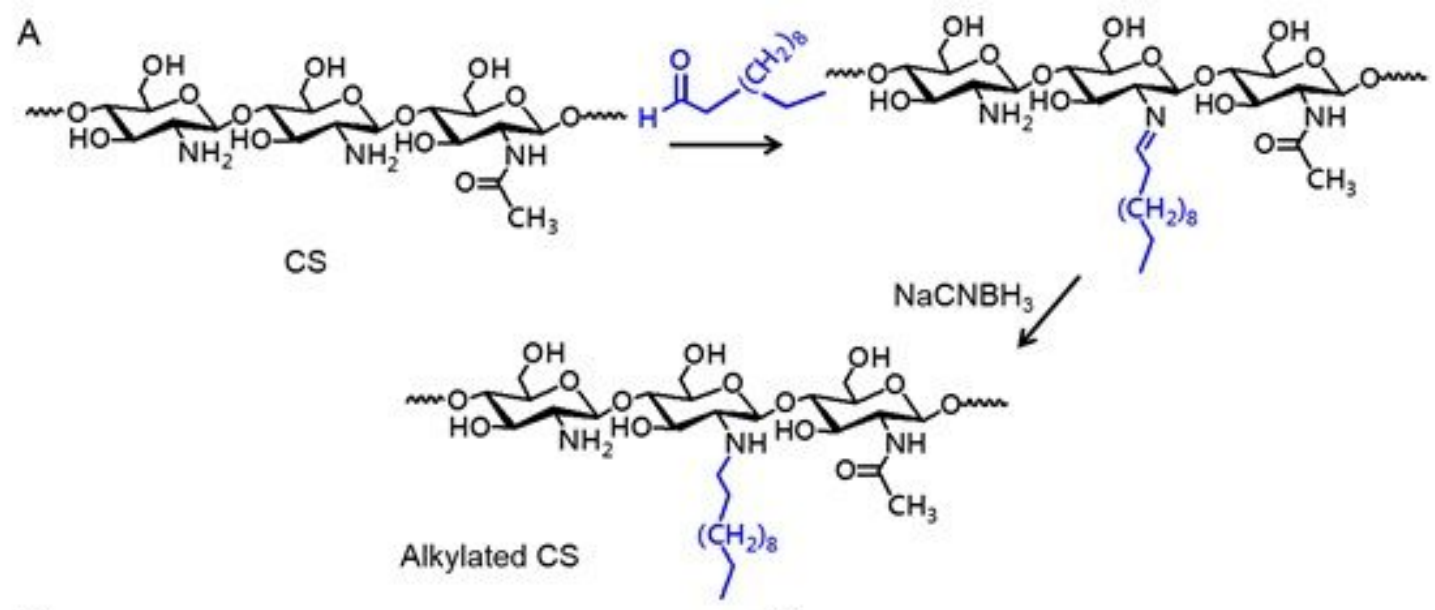

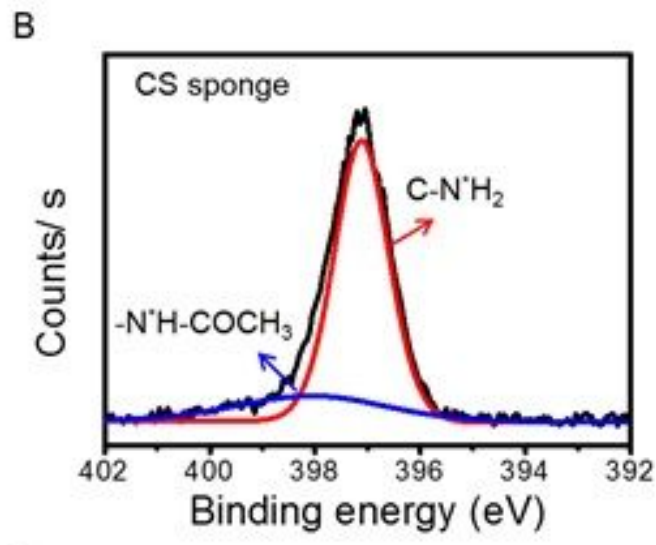

D

Table 1. The area of N1s peak

\begin{tabular}{cccc}
\hline Sponge & $\begin{array}{c}\mathrm{C}-\mathrm{N}^{\prime} \mathrm{H}_{2} \\
\%\end{array}$ & $\begin{array}{c}-\mathrm{N}{ }^{*} \mathrm{H}-\mathrm{COCH}_{3} \\
\%\end{array}$ & $\begin{array}{c}\mathrm{C}-\mathrm{N}{ }^{*}-\mathrm{C} \\
\%\end{array}$ \\
\hline $\mathrm{CS}$ & 82.08 & 17.92 & 0.00 \\
Alkylated CS & 51.05 & 9.11 & 39.87 \\
\hline
\end{tabular}

$\mathrm{C}$

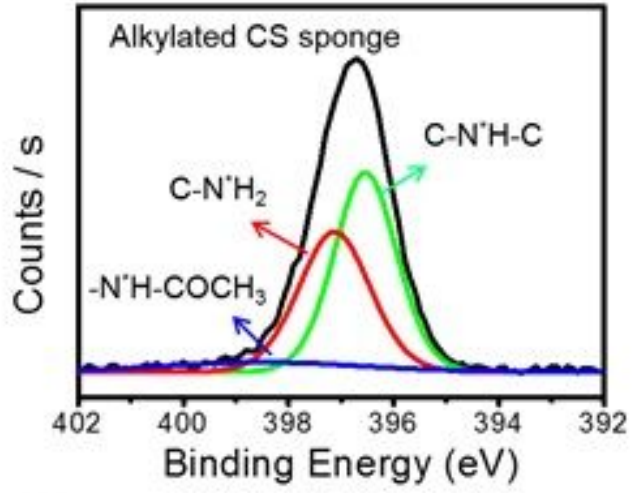

E

Table 2. The calibrate value of $\mathrm{C} 1 \mathrm{~s}$

\begin{tabular}{ccc}
\hline Sponge & $\begin{array}{c}\text { Atomic } \\
\text { Conc \% }\end{array}$ & $\begin{array}{c}\text { Mass } \\
\text { Conc \% }\end{array}$ \\
\hline CS & 46.86 & 39.86 \\
\hline Alkylated CS & 60.16 & 53.67 \\
\hline
\end{tabular}

Figure 2

Chemical characterization of the MACSs. (A) Modification of the CS sponge with DA in the presence of $\mathrm{NaCNBH} 3$ as a reducing agent. (B, C) XPS spectra showing N1s peak of the CS and alkylated CS sponges. (D, E) The area of N1s peak and the calibrate value of C1s in the CS and alkylated CS sponges. 

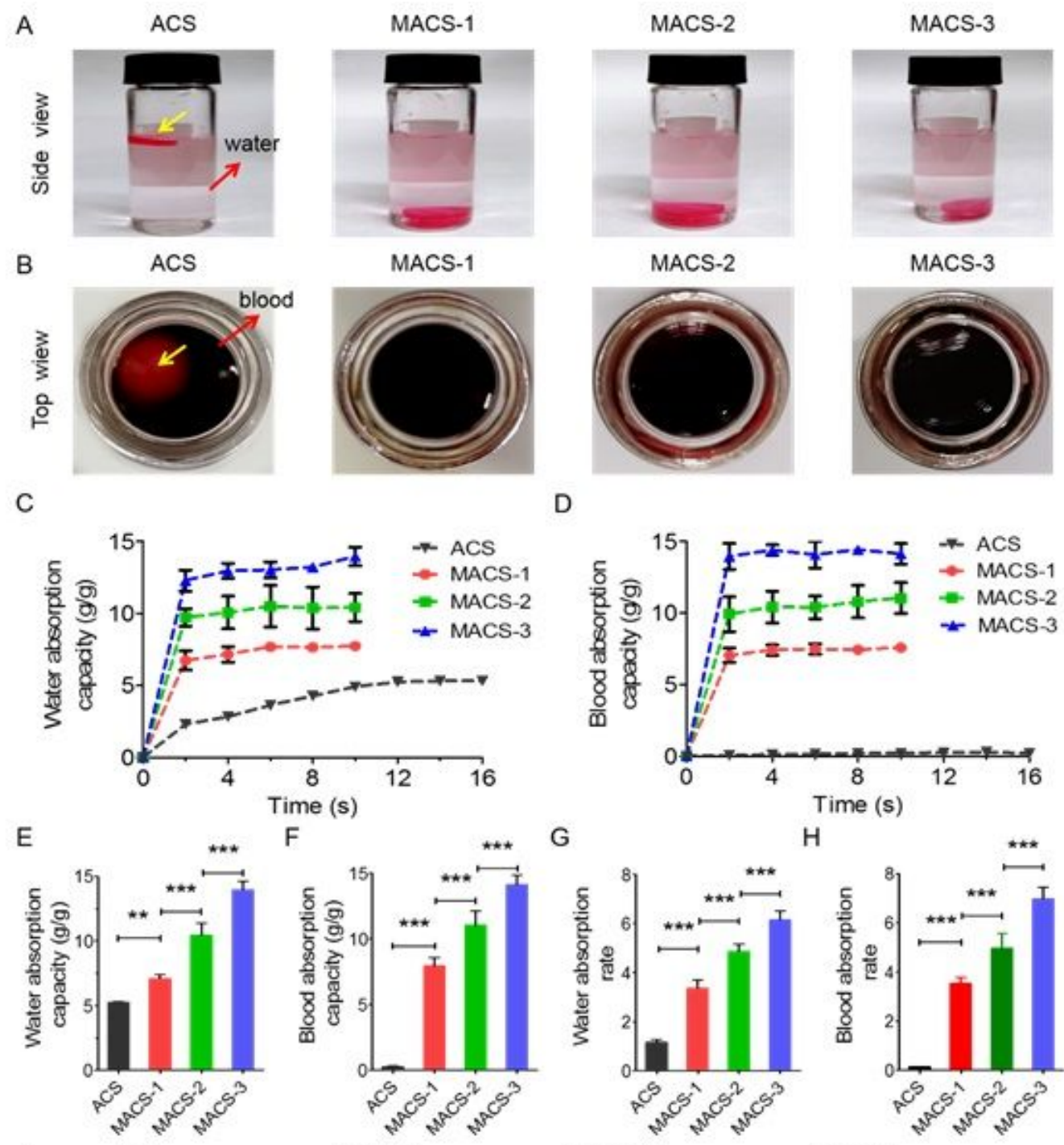

F
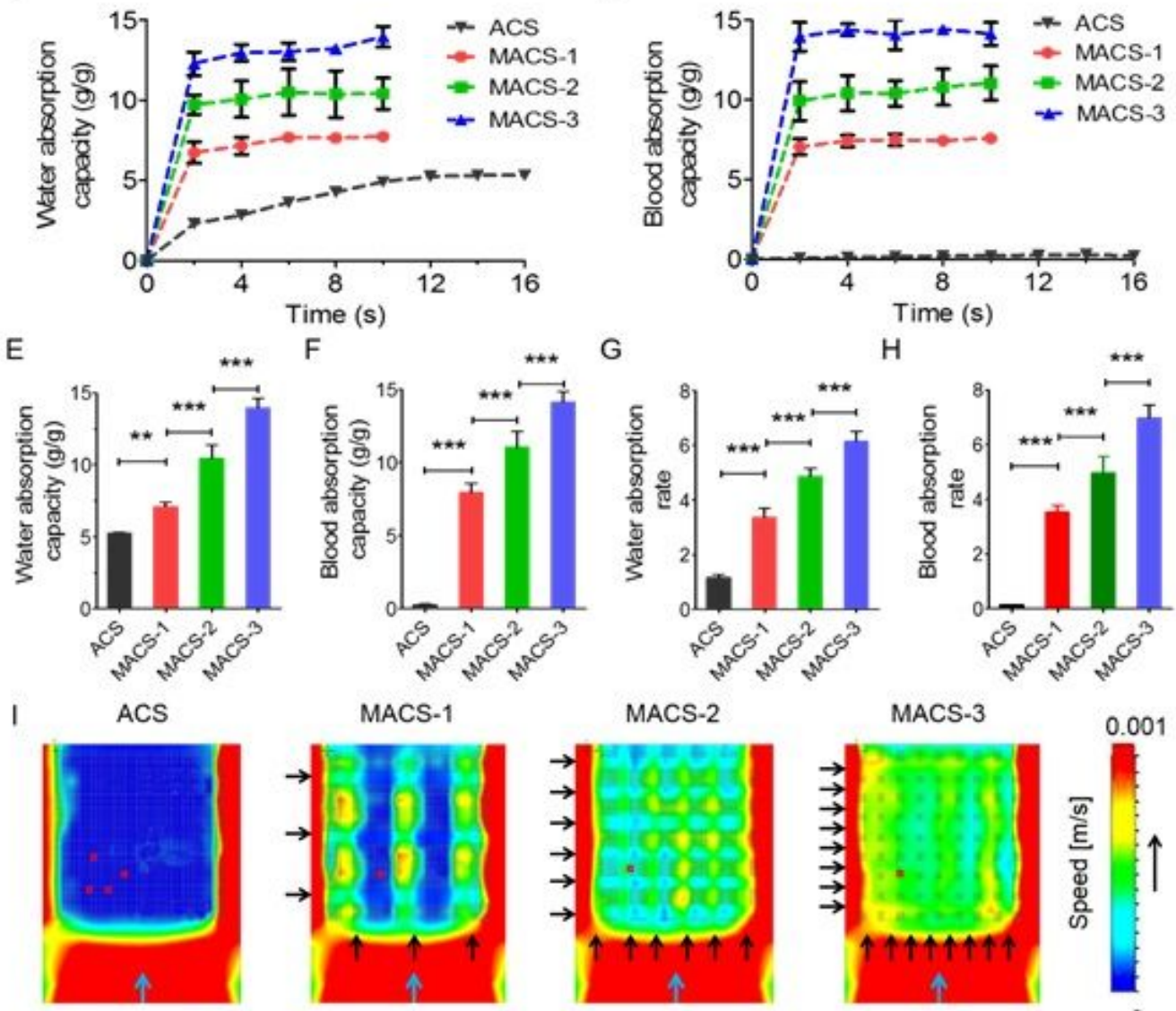

$\square$ : Matrix $\uparrow$ : Flow orientation of fluid
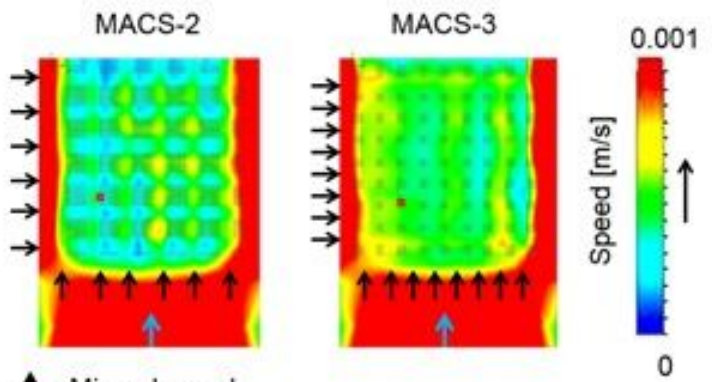

$\mathrm{J}$

Table 3. Total fluid speed $[\mathrm{m} / \mathrm{s}]$

\begin{tabular}{cccc}
\hline ACS & MACS-1 & MACS-2 & MACS-3 \\
\hline 0.0018 & 0.0087 & 0.0125 & 0.0162 \\
\hline
\end{tabular}

\section{Figure 3}

The water/blood absorbability of the ACS and MACSs. (A, B) Photographs of the ACS and MACS-1/2/3 after absorbing water and blood. (C, D) Water and blood absorption capacity-time dynamic curves of the ACS and MACS-1/2/3. (E, F) Maximum water and blood absorption capacity of the ACS and MACS1/2/3. (G, H) Water and blood absorption rate of the ACS and MACS-1/2/3 within 2s. (I) Fluid simulation images of water absorption behaviors of the ACS and MACS-1/2/3. (J) Total fluid speed of the ACS and 
MACS-1/2/3. $n=3$, Data are means \pm SD. ns indicated no significant difference, $* P<0.05, * * P<0.01$, $\star * * P<0.001$.
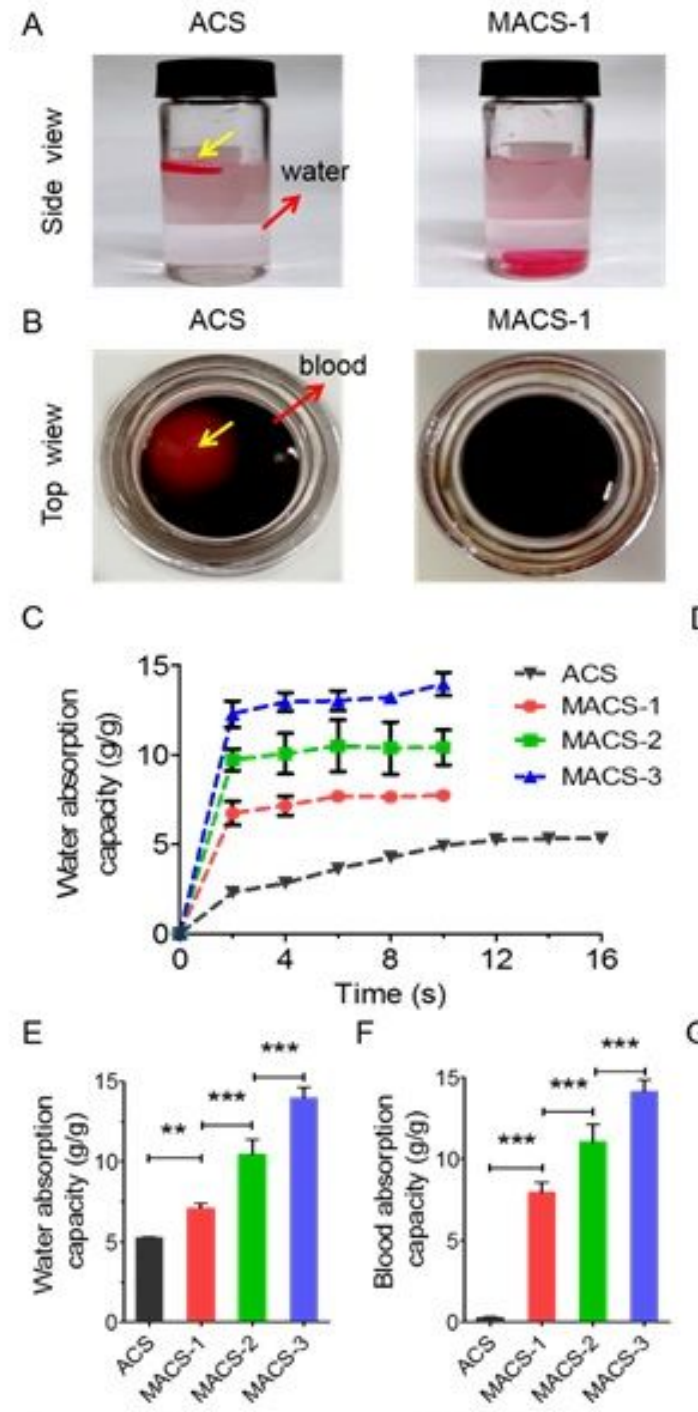

I

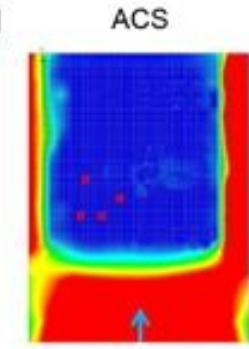

口: Matrix $\uparrow$ : Flow orientation of fluid

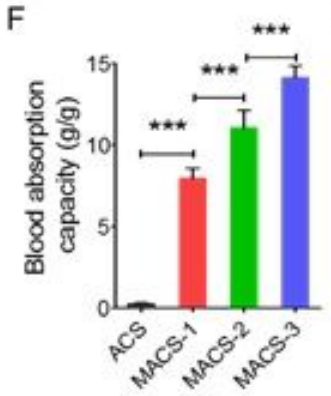

MACS-1

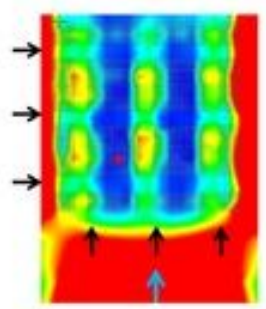

Table 3.
MACS-2

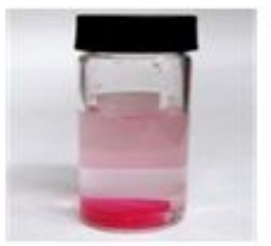

MACS-2

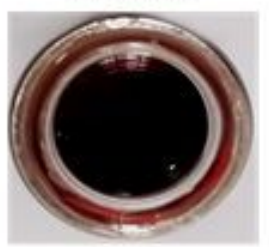

MACS-3

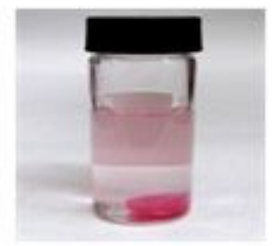

MACS-3

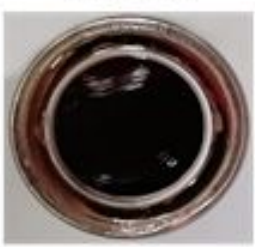

D

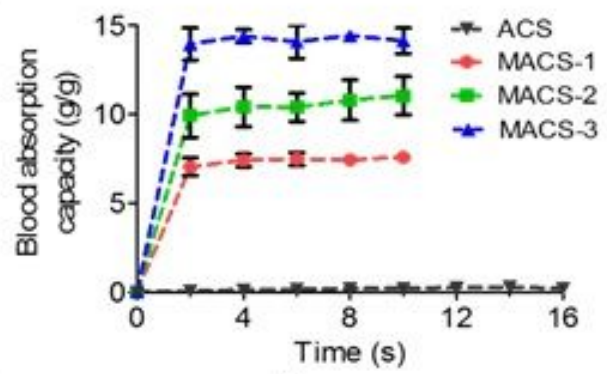

G $\quad H$

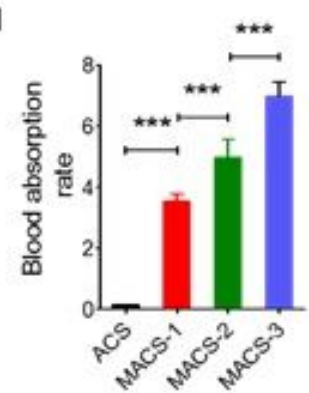

$\mathrm{J}$

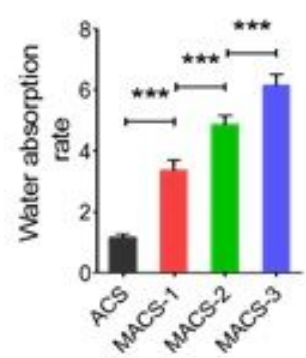

MACS-3
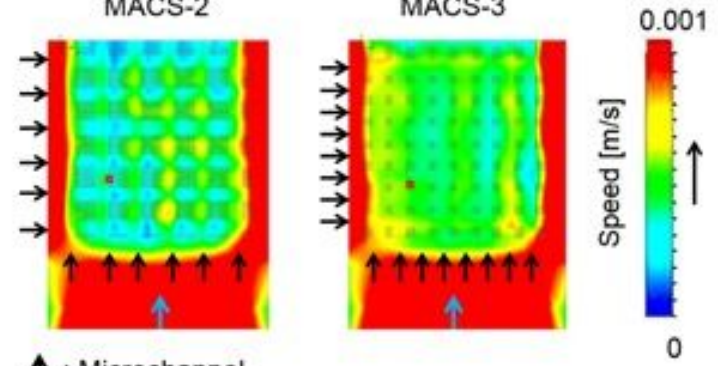

$\uparrow:$ Microchannel

\begin{tabular}{cccc}
\hline ACS & MACS-1 & MACS-2 & MACS-3 \\
\hline 0.0018 & 0.0087 & 0.0125 & 0.0162 \\
\hline
\end{tabular}

Figure 3

The water/blood absorbability of the ACS and MACSs. (A, B) Photographs of the ACS and MACS- $1 / 2 / 3$ after absorbing water and blood. (C, D) Water and blood absorption capacity-time dynamic curves of the ACS and MACS-1/2/3. (E, F) Maximum water and blood absorption capacity of the ACS and MACS- 
1/2/3. (G, H) Water and blood absorption rate of the ACS and MACS-1/2/3 within 2s. (I) Fluid simulation images of water absorption behaviors of the ACS and MACS-1/2/3. (J) Total fluid speed of the ACS and MACS-1/2/3. $n=3$, Data are means \pm SD. ns indicated no significant difference, ${ }^{*} P<0.05,{ }^{\star \star} P<0.01$, $* * * P<0.001$.

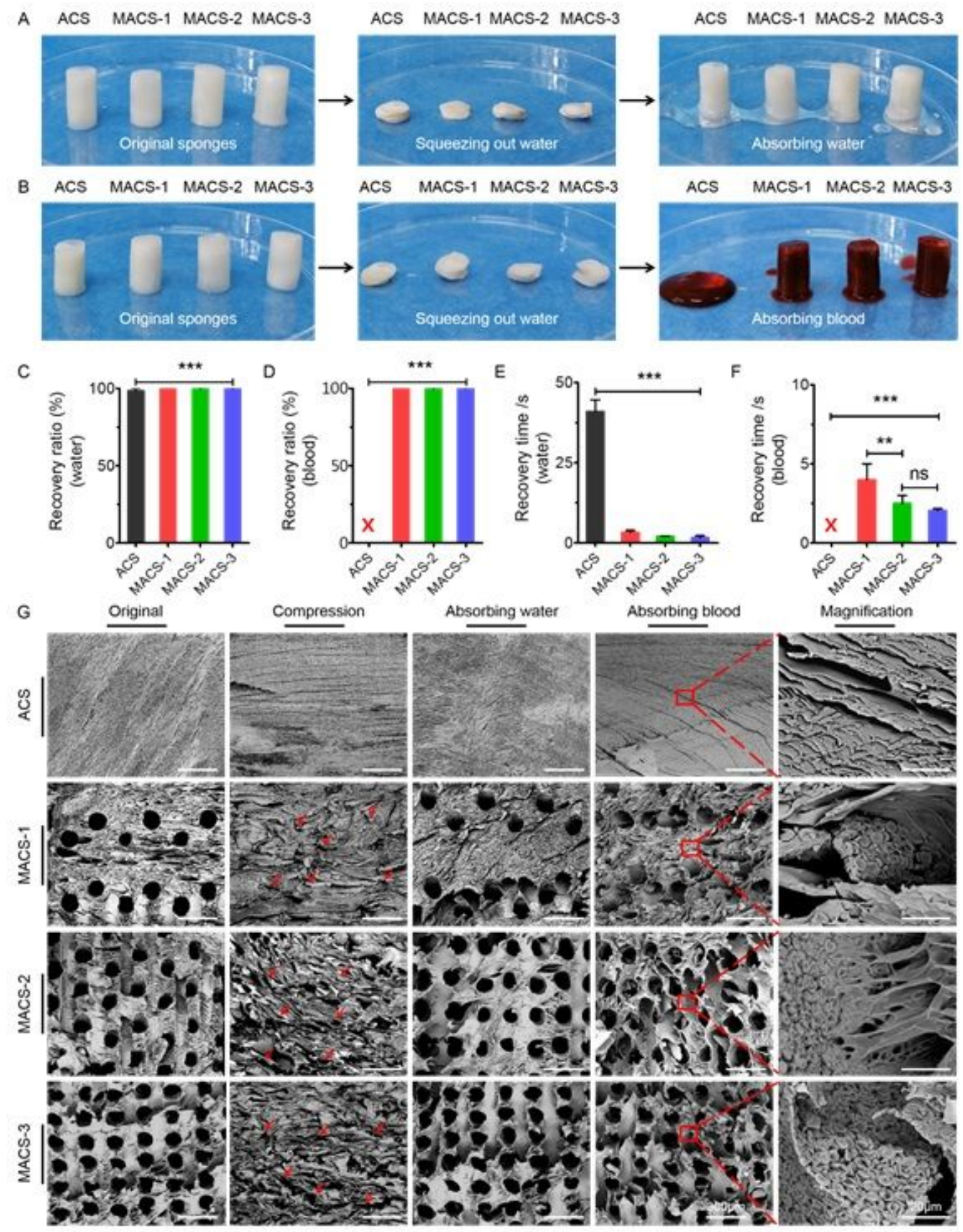

H Table 4. Comparsion of shape recovery time between the MACS-2/3 and reported shape memory hemostats after absorbing water and blood

\begin{tabular}{|c|c|c|c|c|c|c|c|}
\hline & & & & [1 & {$[6]$} & [16] & \\
\hline Hemostats & XStat $^{\mathrm{TM}}$ & GT25/DA8 & $\mathrm{H} 40^{\circ}$ & Peanut & TRAP/Sp & QCS/PDA4 & MACS-2/3 \\
\hline Water & $20 \mathrm{~s}$ & $4.2 \pm 0.2 \mathrm{~s}$ & $4 \mathrm{~min}$ & $\sim 10 \mathrm{~s}$ & $10 \mathrm{~s}$ & $2.4 \mathrm{~s}$ & $2.0 \pm 0.1 \mathrm{~s}$ \\
\hline Blood & $20 \mathrm{~s}$ & $23.4 \pm 2.4 \mathrm{~s}$ & & $\sim 10 \mathrm{~s}$ & & $19.8 \pm 4.9 \mathrm{~s}$ & $2.5 \pm 0.5 \mathrm{~s}$ \\
\hline
\end{tabular}

Figure 4 
The shape-memory property of the ACS and MACSs after absorbing water and blood. (A, B) Photographs of the water- and blood-triggered shape recovery of the ACS and MACS-1/2/3. (C, D, E, F) The shaperecovery ratio and time of the compressed sponges. (G) SEM images showing the microstructure of the compressed sponges before and after absorbing water and blood. Red arrows represented the flat channels. $(\mathrm{H})$ Comparison of shape-recovery time between the MACS-2/3 and reported shape-memory hemostats. $n=3$, Data are means \pm SD. ns indicated no significant difference, ${ }^{*} P<0.05,{ }^{\star} * P<0.01$, $\star \star \star P<0.001$.

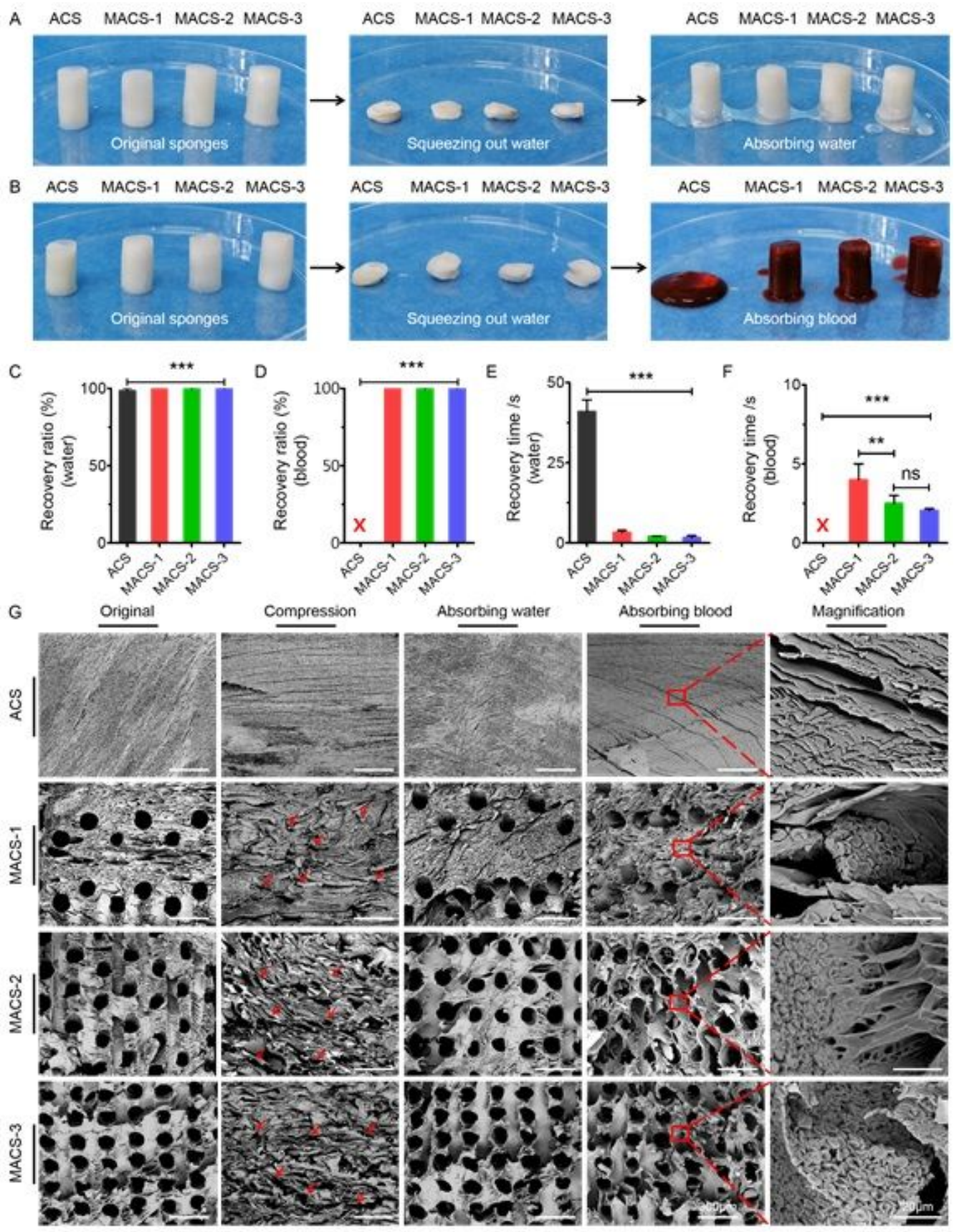

H Table 4. Comparsion of shape recovery time between the MACS-2/3 and reported shape memory hemostats after absorbing water and blood

\begin{tabular}{|c|c|c|c|c|c|c|c|}
\hline & & & [15] & & 이 [6] & {$[16]$} & \\
\hline Hemostats & XStat ${ }^{\mathrm{TM}}$ & GT25/DA8 & $\mathrm{H} 40$ & Peanut & TRAP/Sp & QCS/PDA4 & MACS $-2 / 3$ \\
\hline Water & $20 \mathrm{~s}$ & $4.2 \pm 0.2 \mathrm{~s}$ & $4 \mathrm{~min}$ & $\sim 10 \mathrm{~s}$ & $10 \mathrm{~s}$ & $2.4 \mathrm{~s}$ & $2.0 \pm 0.1 \mathrm{~s}$ \\
\hline Blood & $20 \mathrm{~s}$ & $23.4 \pm 2.4 \mathrm{~s}$ & & $\sim 10 \mathrm{~s}$ & & $19.8 \pm 4.9 \mathrm{~s}$ & $2.5 \pm 0.5 \mathrm{~s}$ \\
\hline
\end{tabular}




\section{Figure 4}

The shape-memory property of the ACS and MACSs after absorbing water and blood. (A, B) Photographs of the water- and blood-triggered shape recovery of the ACS and MACS-1/2/3. (C, D, E, F) The shaperecovery ratio and time of the compressed sponges. (G) SEM images showing the microstructure of the compressed sponges before and after absorbing water and blood. Red arrows represented the flat channels. $(\mathrm{H})$ Comparison of shape-recovery time between the MACS-2/3 and reported shape-memory hemostats. $n=3$, Data are means $\pm S D$. ns indicated no significant difference, ${ }^{*} P<0.05,{ }^{\star} * P<0.01$, $* * * P<0.001$.

A
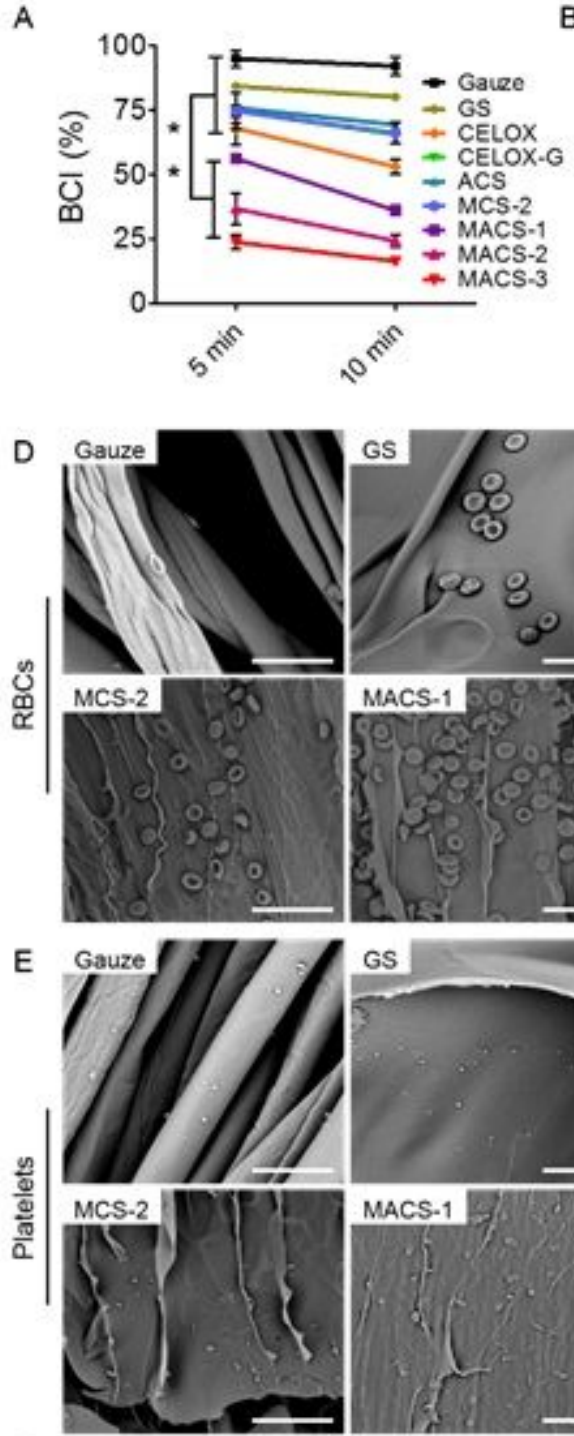

$\mathrm{F}$
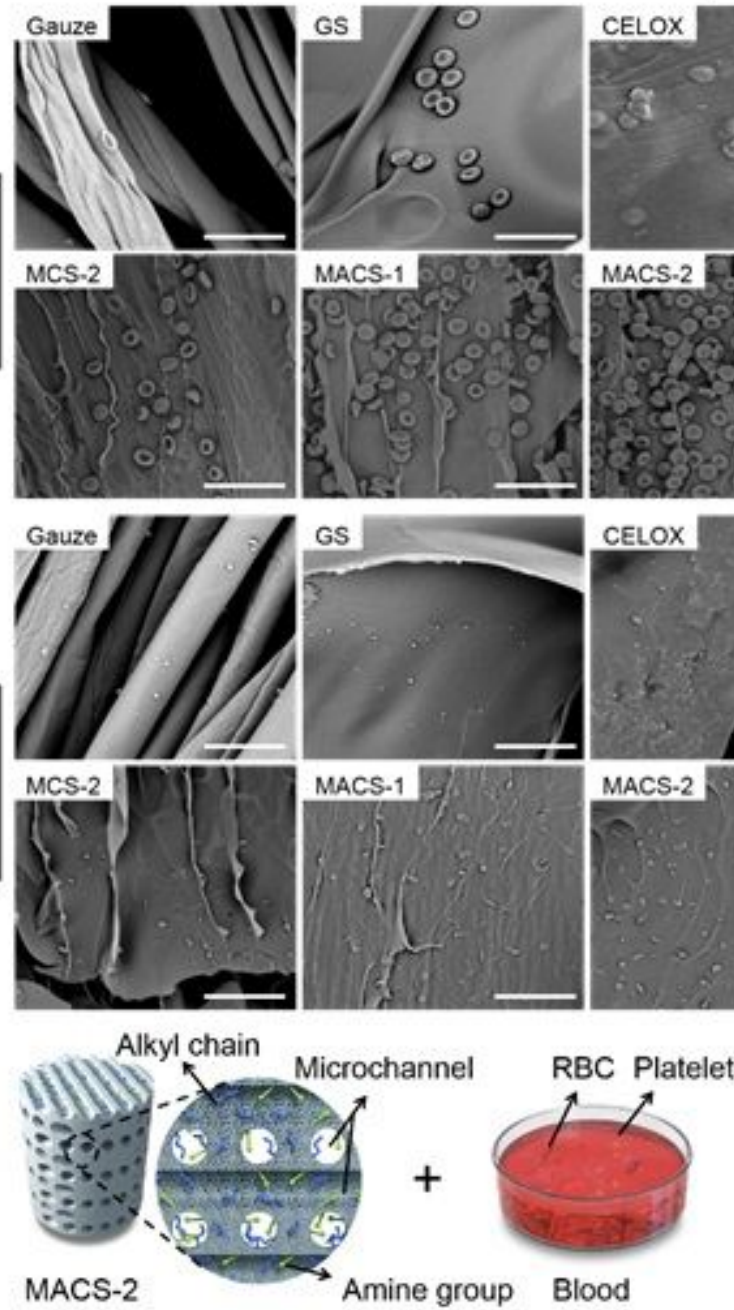
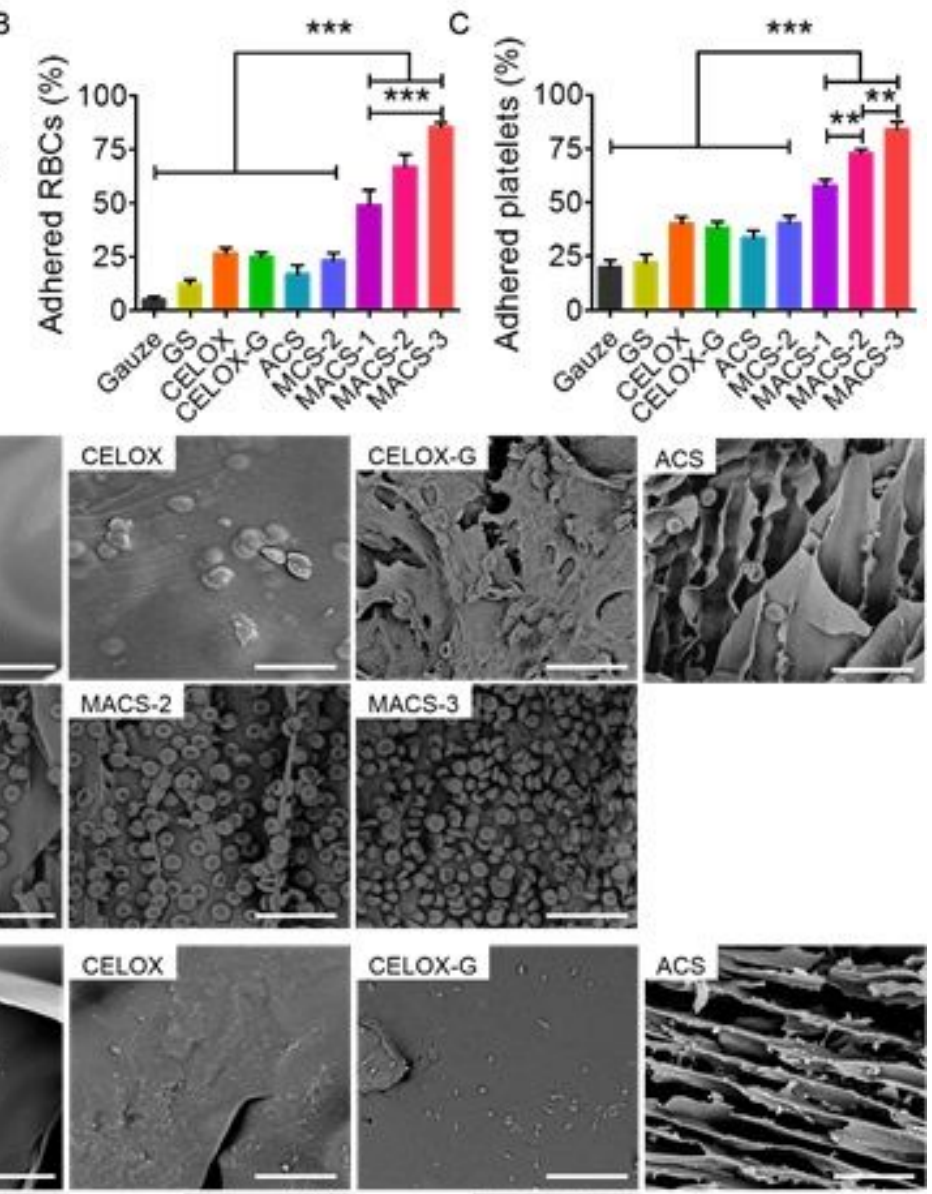

MACS-3
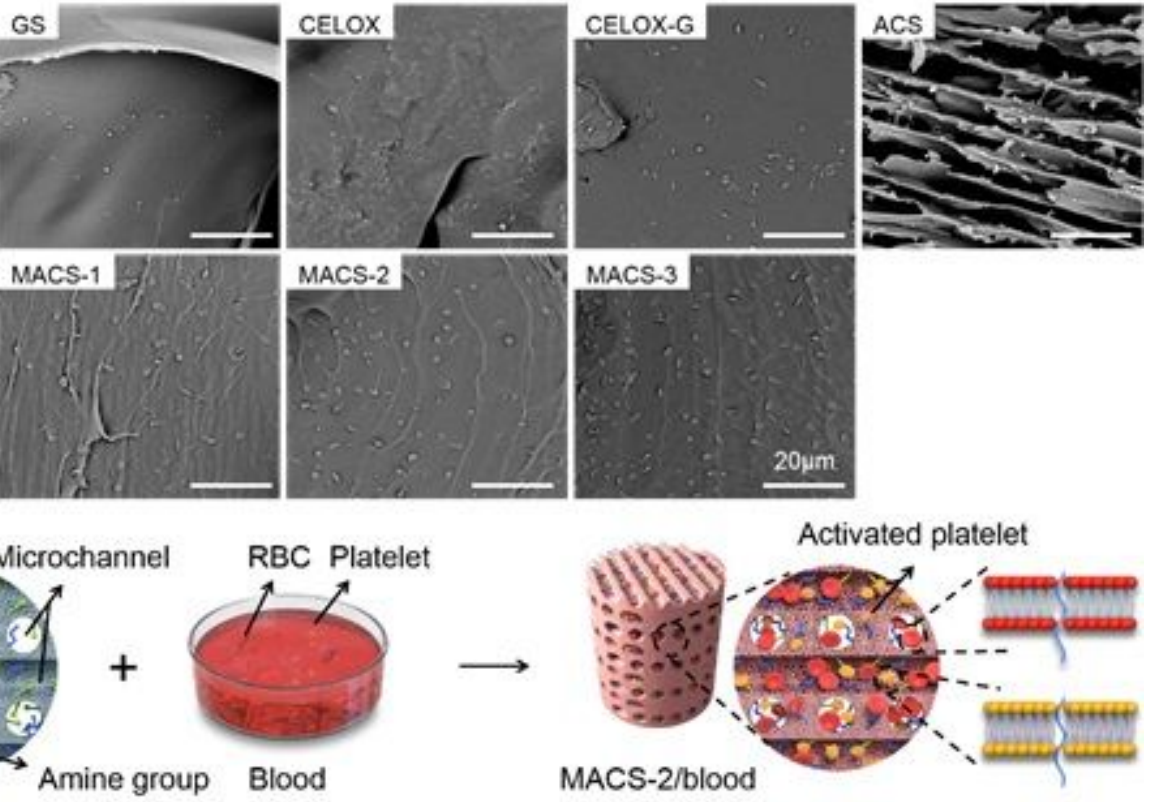

Figure 5 
The pro-coagulant ability of the gauze, GS, CELOX, CELOX-G, ACS, MCS-2, and MACSs. (A) The BCl-time curves of various samples. $(B, C)$ The number of adhered RBCs and platelets on various samples. $(D, E)$ SEM images showing adhesion of RBCs and platelets on various samples. (F) Schematic diagram illustrating the pro-coagulant mechanism of the MACSs. $n=3$, Data are means $\pm S D$. ns indicated no significant difference, ${ }^{*} \mathrm{P}<0.01,{ }^{*} \mathrm{P}<0.01,{ }^{*} * \mathrm{P}<0.001$.
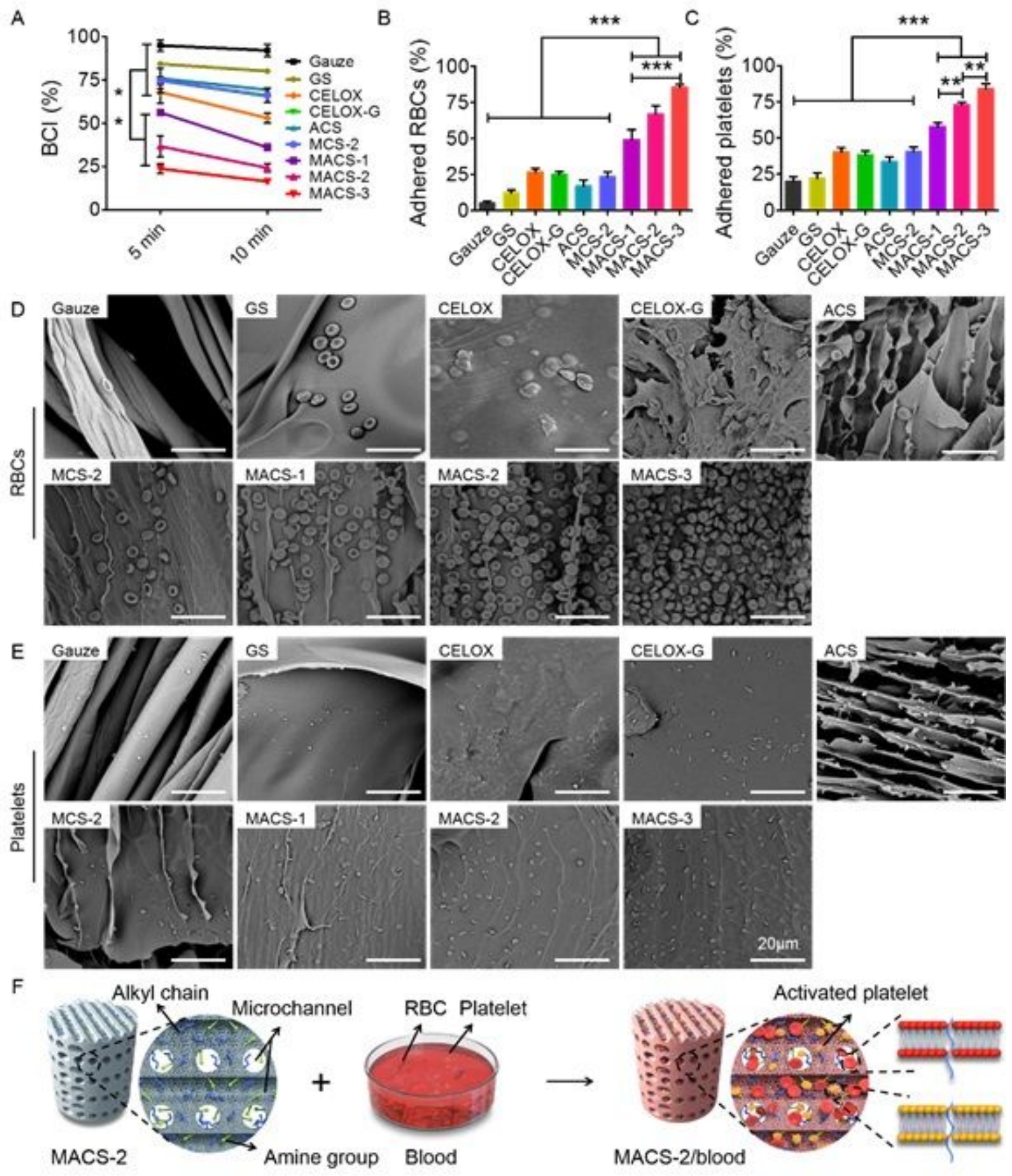

Figure 5

The pro-coagulant ability of the gauze, GS, CELOX, CELOX-G, ACS, MCS-2, and MACSs. (A) The BCl-time curves of various samples. $(B, C)$ The number of adhered $R B C s$ and platelets on various samples. $(D, E)$ SEM images showing adhesion of RBCs and platelets on various samples. $(F)$ Schematic diagram 
illustrating the pro-coagulant mechanism of the MACSs. $n=3$, Data are means \pm SD. ns indicated no significant difference, ${ }^{*} P<0.01, * * P<0.01, * * * P<0.001$.

A

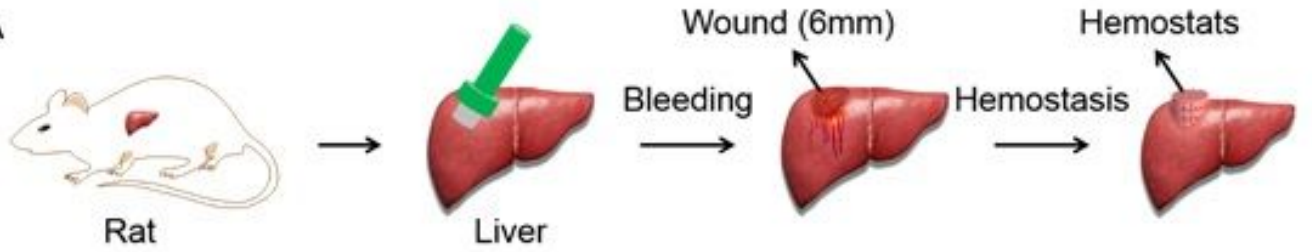

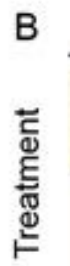

Blank
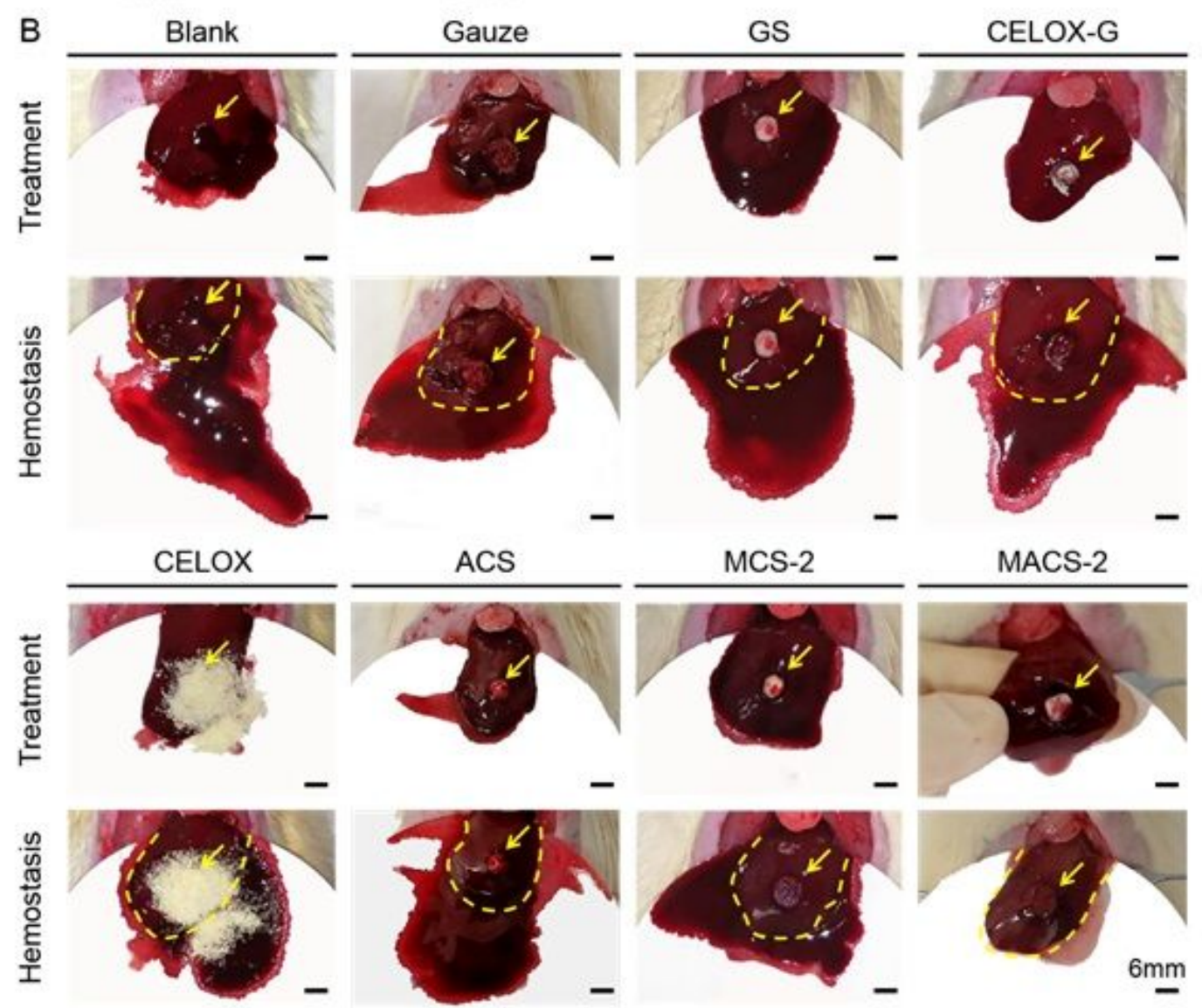

C
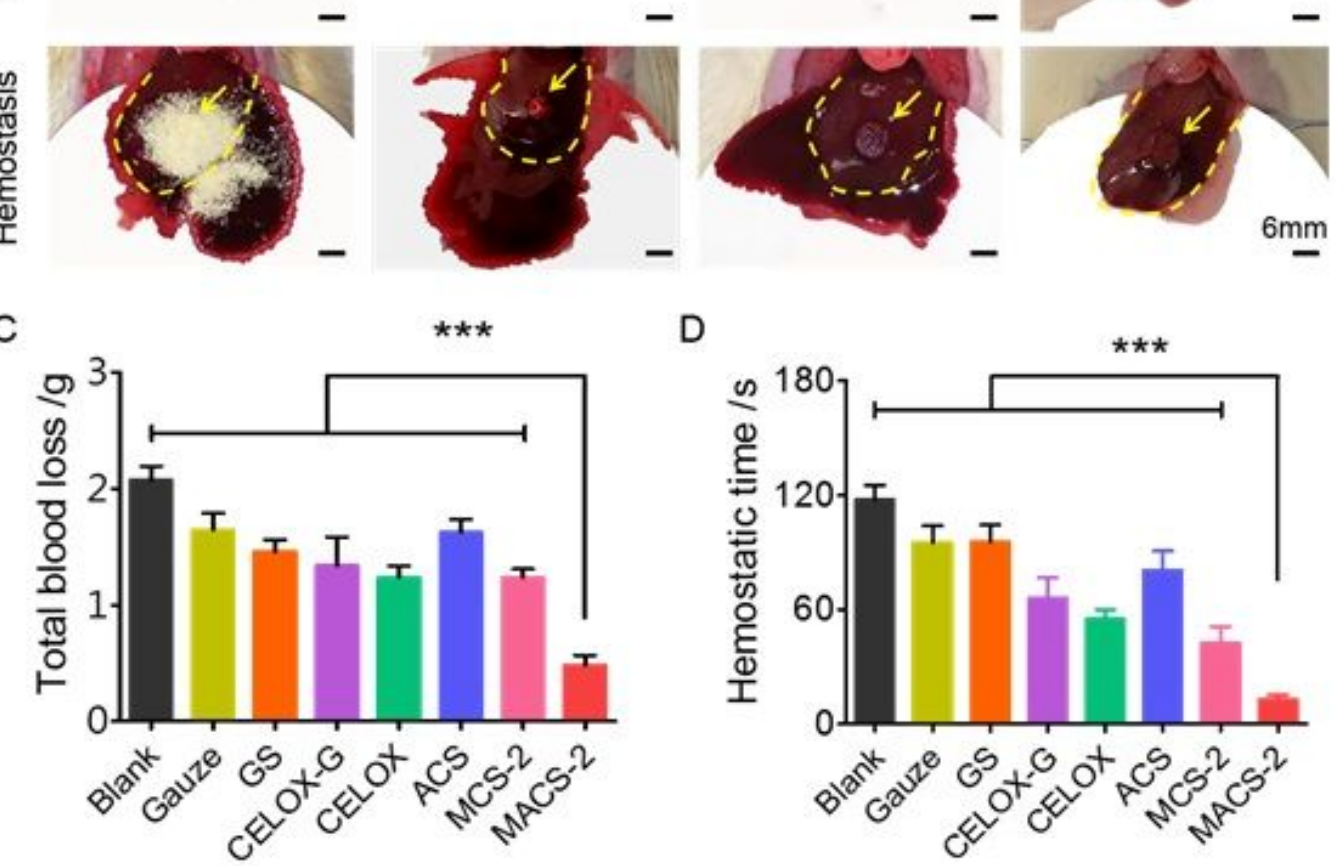

Figure 6

Hemostasis in the normal rat liver perforation wound model. (A) Schematic illustration of the hemostatic process of hemostats in a rat liver perforation wound model. (B) Photographs of the hemostatic effect of the gauze, GS, CELOX-G, CELOX, ACS, MCS-2, and MACS-2. The yellow arrow represents the bleeding site. 
The yellow dotted line represents the boundary of the liver. (C, D) Total blood loss and hemostatic time in the gauze, GS, CELOX-G, CELOX, ACS, MCS-2, and MACS-2 groups. $n=3$, Data are means \pm SD. ns indicated no significant difference, ${ }^{*} P<0.01,{ }^{*} \mathrm{P}<0.01,{ }^{* * *} \mathrm{P}<0.001$.

A

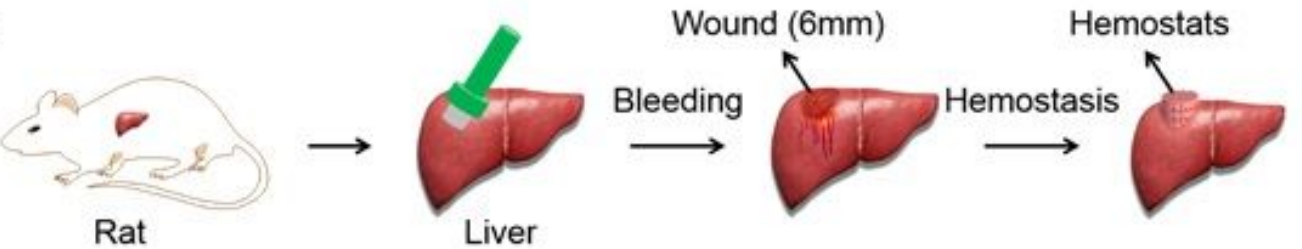

B

Blank

壱
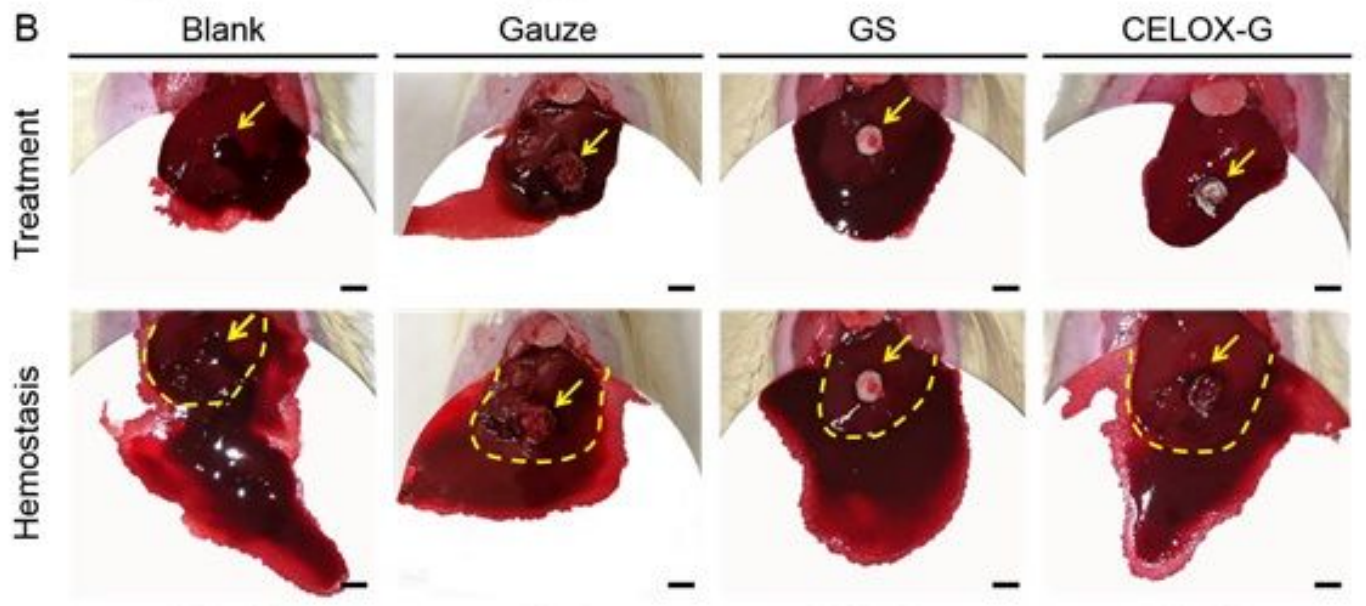

-
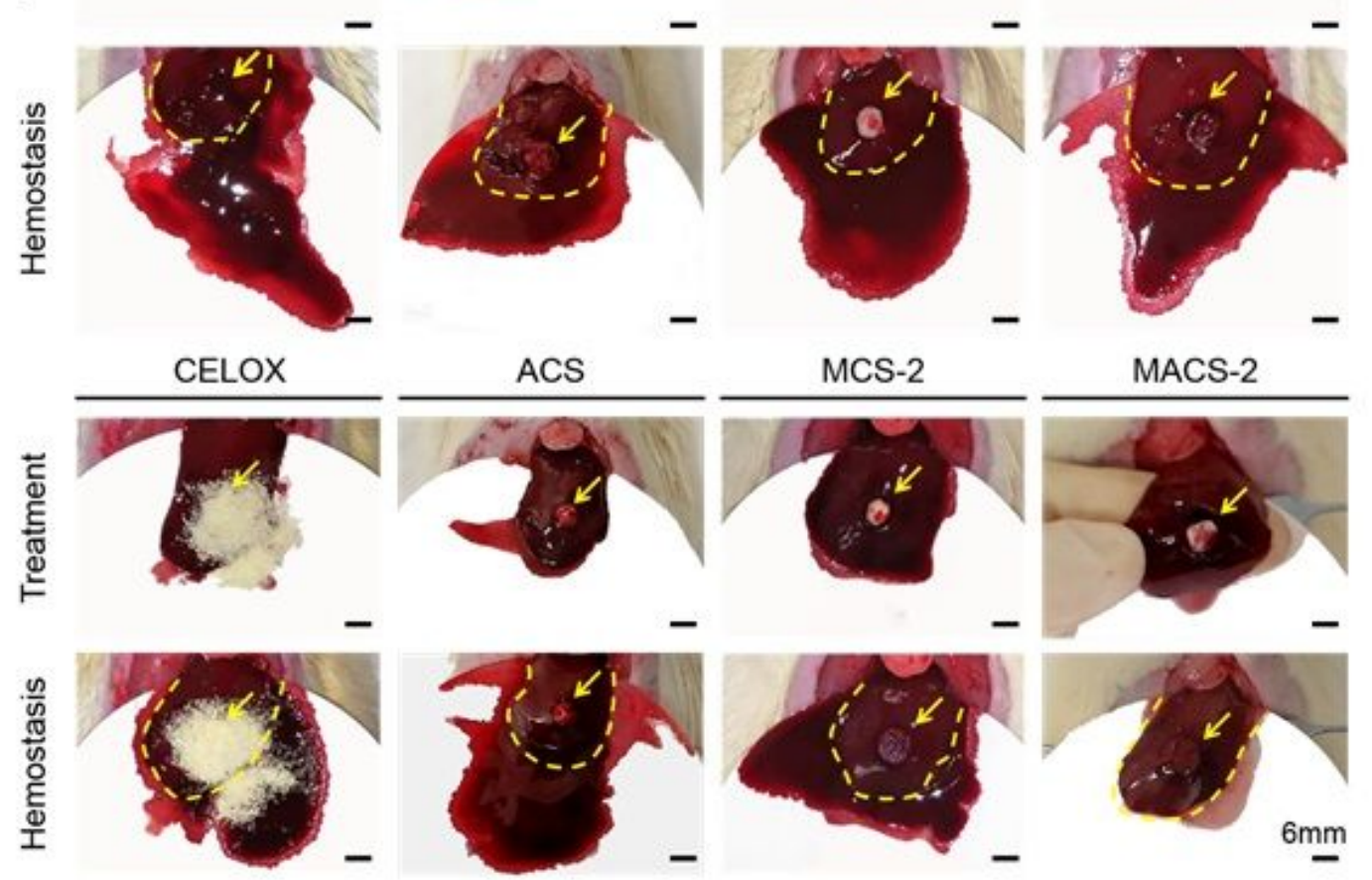

C

$\star * *$

D
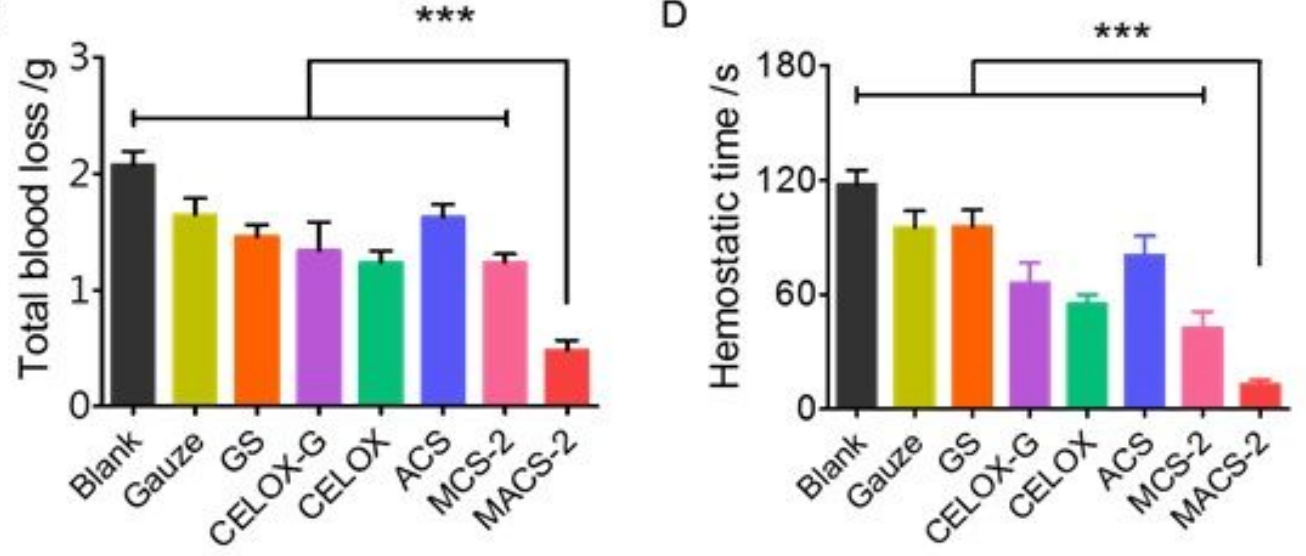

Figure 6

Hemostasis in the normal rat liver perforation wound model. (A) Schematic illustration of the hemostatic process of hemostats in a rat liver perforation wound model. (B) Photographs of the hemostatic effect of 
the gauze, GS, CELOX-G, CELOX, ACS, MCS-2, and MACS-2. The yellow arrow represents the bleeding site. The yellow dotted line represents the boundary of the liver. (C, D) Total blood loss and hemostatic time in the gauze, GS, CELOX-G, CELOX, ACS, MCS-2, and MACS-2 groups. $n=3$, Data are means \pm SD. ns indicated no significant difference, ${ }^{*} P<0.01, * * P<0.01, * \star * P<0.001$.

A
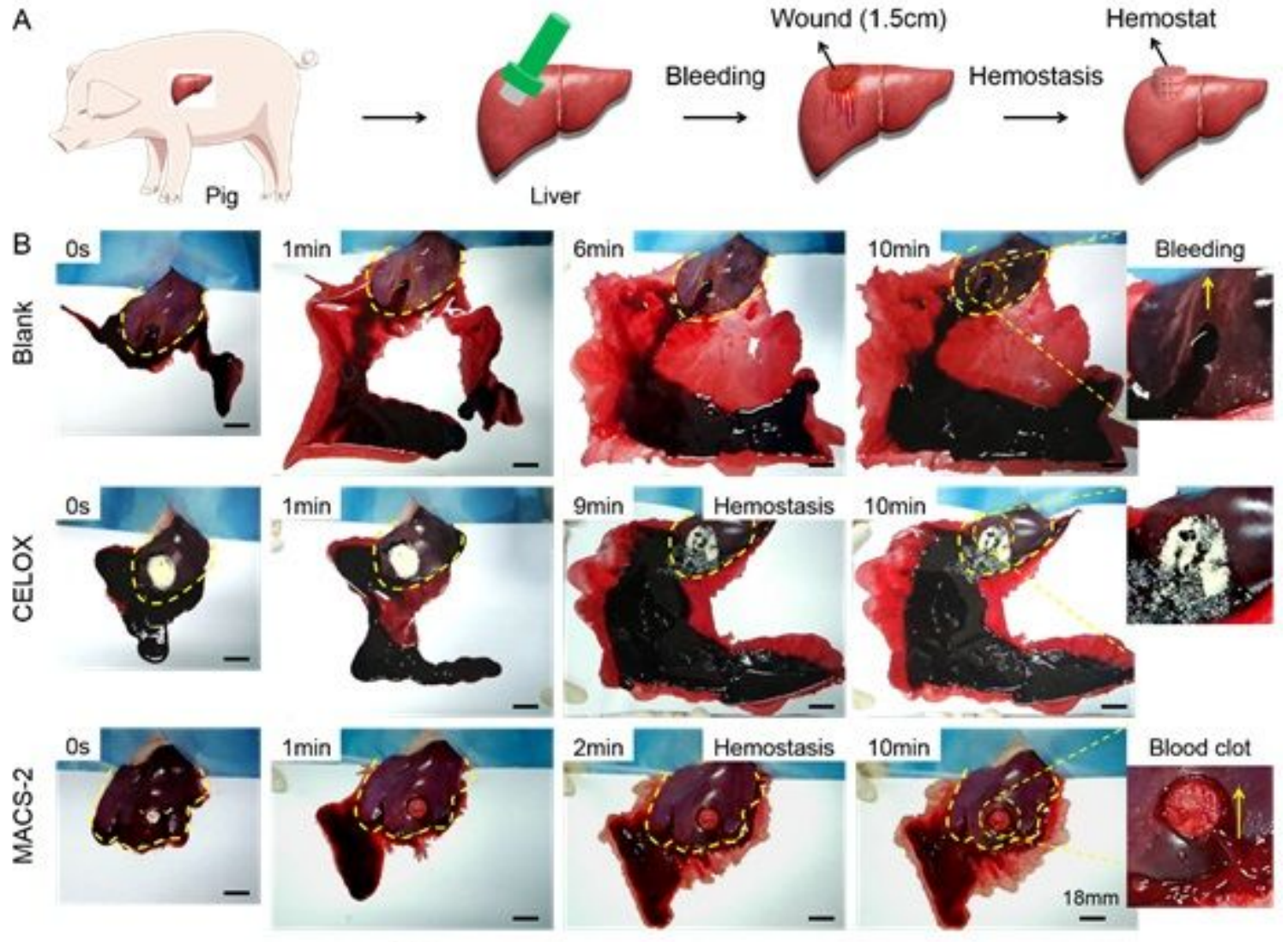

C

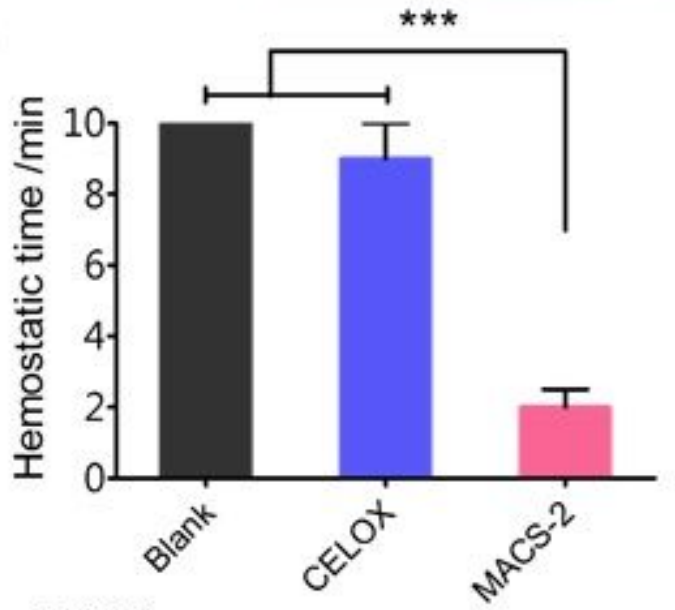

E MACS-2

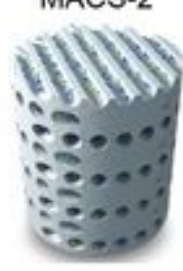

Squeezing out water
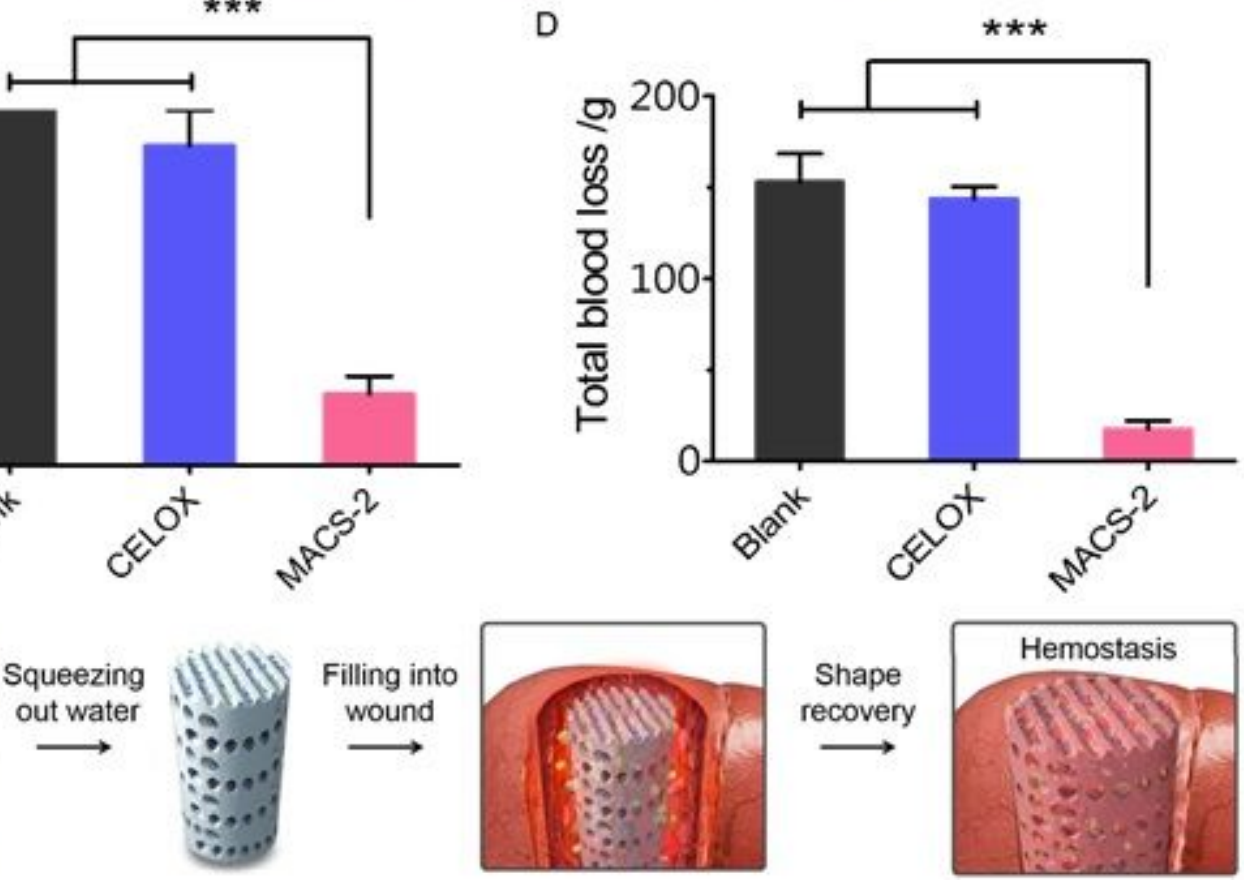

Figure 7 
Hemostasis in a lethal pig liver perforation wound model. (A) Schematic illustration of the hemostatic process of hemostats. (B) Photographs of the hemostatic effect of the blank, CELOX, and MACS-2 groups. The yellow dotted line represents the boundary of the liver. (C, D) Hemostatic time and total blood loss in the blank, CELOX, and MACS-2 groups. (E) Schematic diagram of hemostatic procedure and mechanism of the MACS-2. $n=3$, Data are means \pm SD. ns indicated no significant difference, ${ }^{*}<0.01$, $* * \mathrm{P}<0.01, * * * \mathrm{P}<0.001$.

A
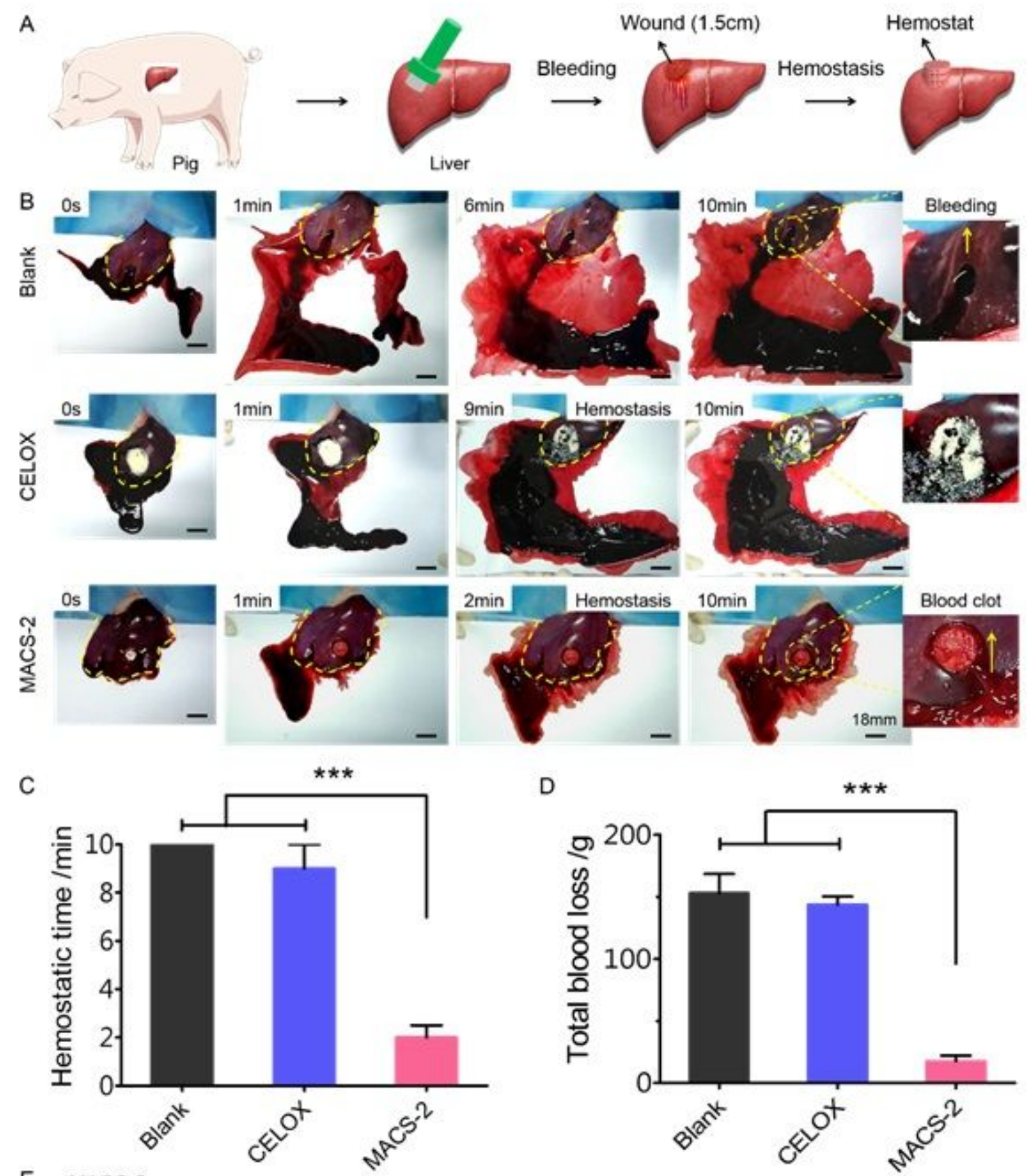

E MACS-2
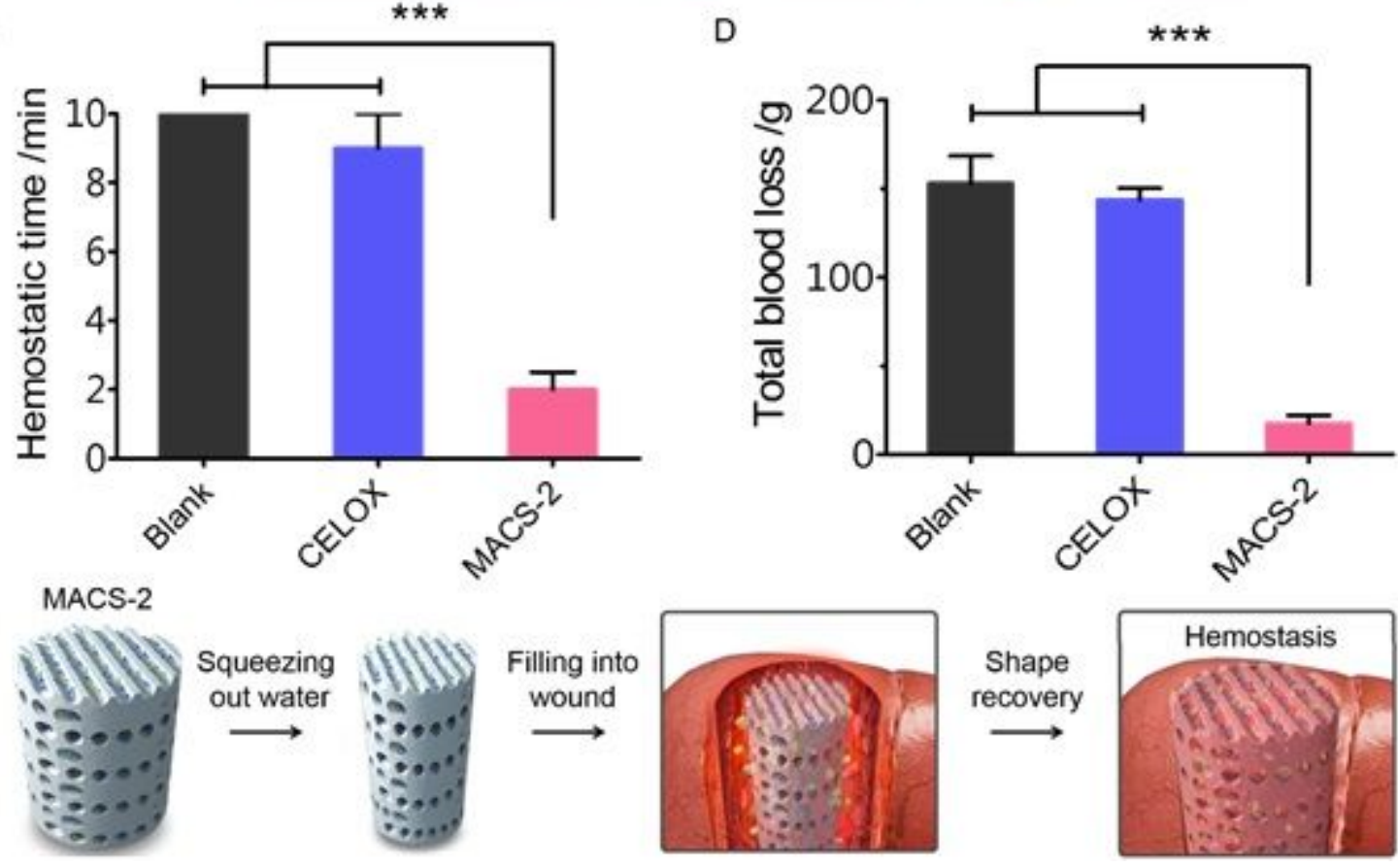

Figure 7 
Hemostasis in a lethal pig liver perforation wound model. (A) Schematic illustration of the hemostatic process of hemostats. (B) Photographs of the hemostatic effect of the blank, CELOX, and MACS-2 groups. The yellow dotted line represents the boundary of the liver. (C, D) Hemostatic time and total blood loss in the blank, CELOX, and MACS-2 groups. (E) Schematic diagram of hemostatic procedure and mechanism of the MACS-2. $n=3$, Data are means \pm SD. ns indicated no significant difference, ${ }^{*}<<0.01$, $* * \mathrm{P}<0.01, * * * \mathrm{P}<0.001$.

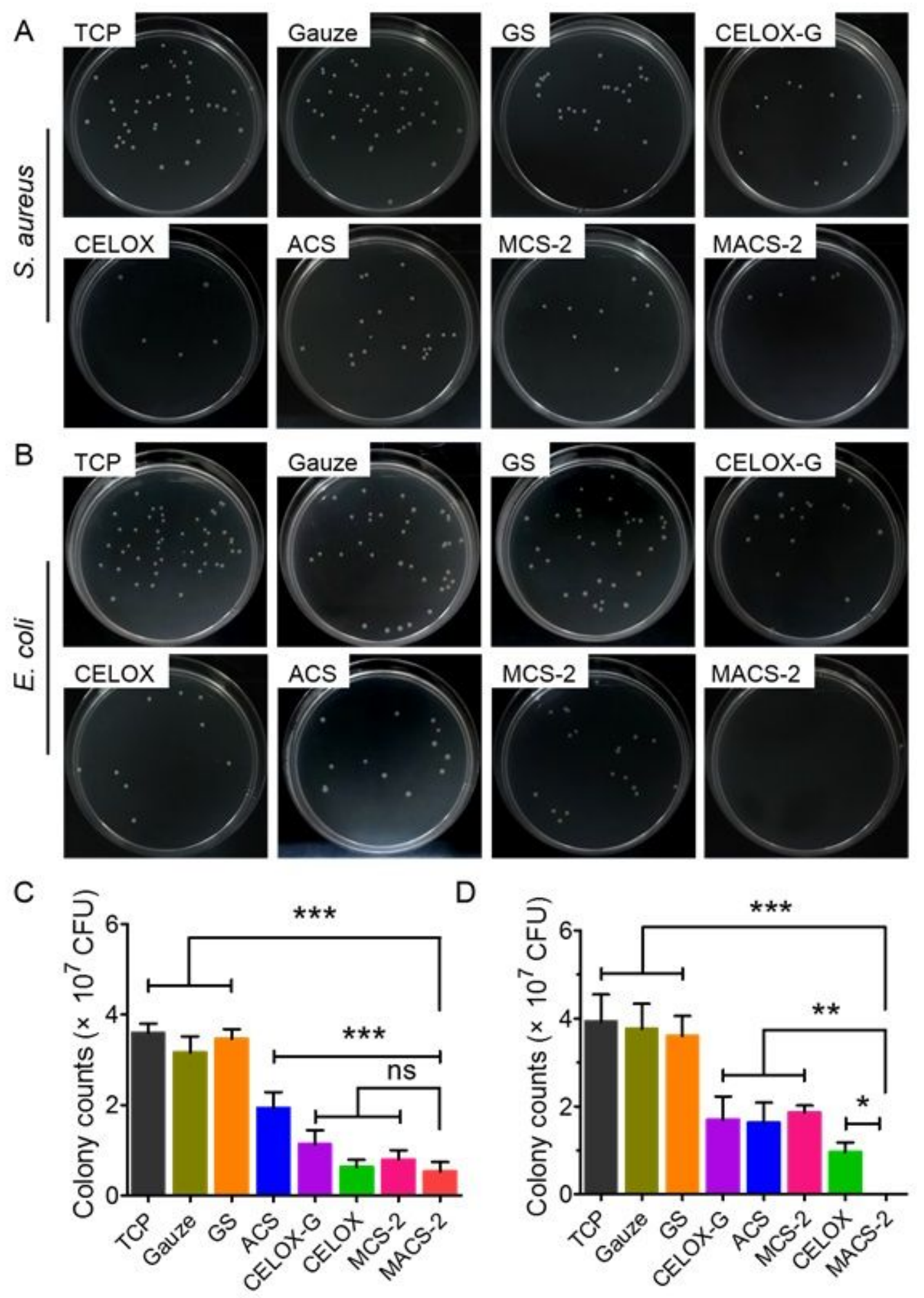

Figure 8 
In vitro anti-infective property of the MACS-2 and other hemostats. (A, B) Photographs of CFUs of S. aureus and E. coli grown on LB agar plates after contacting with TCP, gauze, GS, CELOX-G, CELOX, ACS, MCS-2, and MACS-2, respectively. (C, D) Corresponding statistical results of the CFUs of S. aureus and E. coli. $n=3$, Data are means \pm SD. ns indicated no significant difference, ${ }^{*} P<0.05,{ }^{*} P<0.01,{ }^{* \star *} P<0.001$.

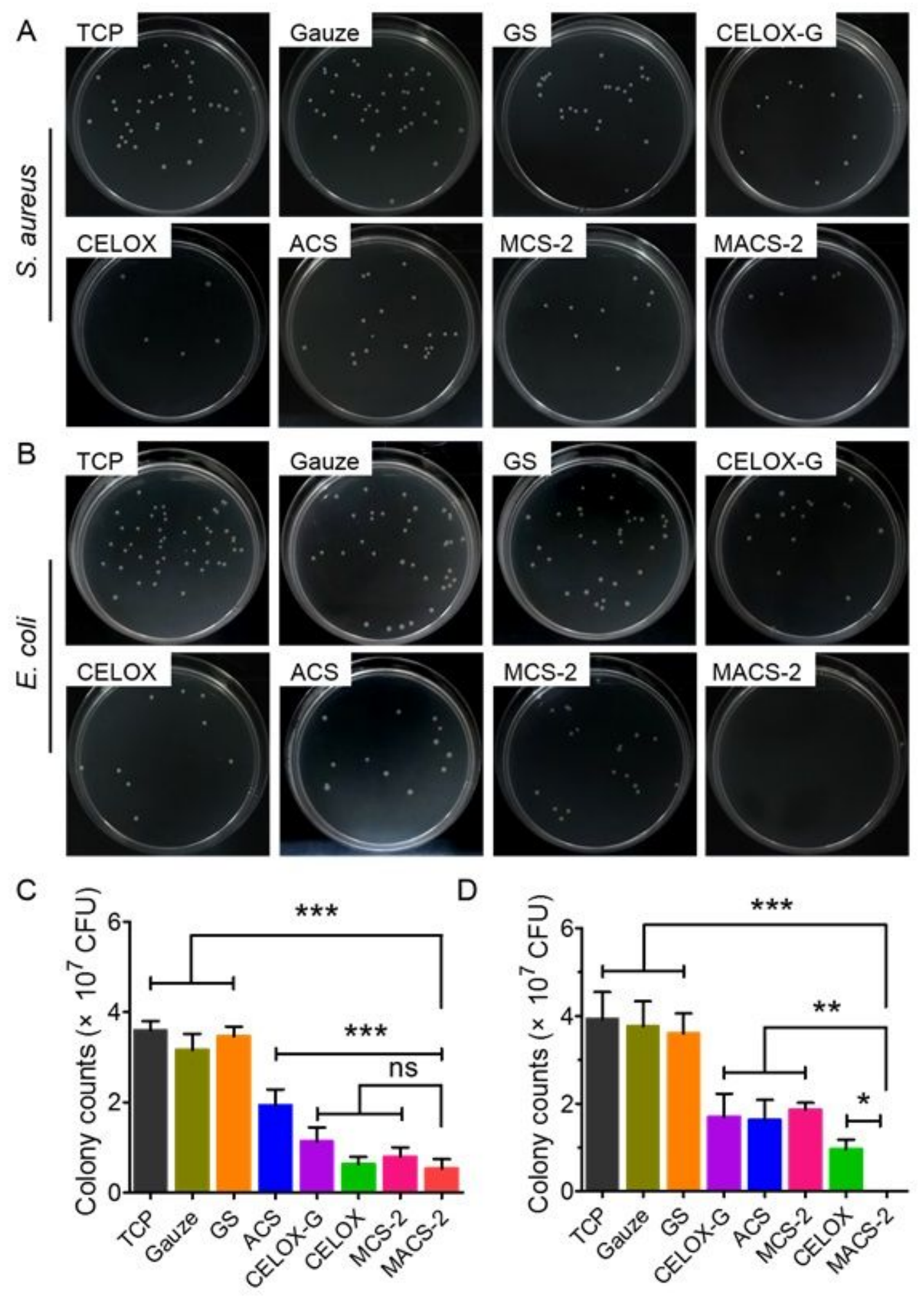

Figure 8 
In vitro anti-infective property of the MACS-2 and other hemostats. (A, B) Photographs of CFUs of S. aureus and E. coli grown on LB agar plates after contacting with TCP, gauze, GS, CELOX-G, CELOX, ACS, MCS-2, and MACS-2, respectively. (C, D) Corresponding statistical results of the CFUs of S. aureus and E. coli. $n=3$, Data are means \pm SD. ns indicated no significant difference, ${ }^{*} P<0.05,{ }^{*} P<0.01,{ }^{*} * * P<0.001$.

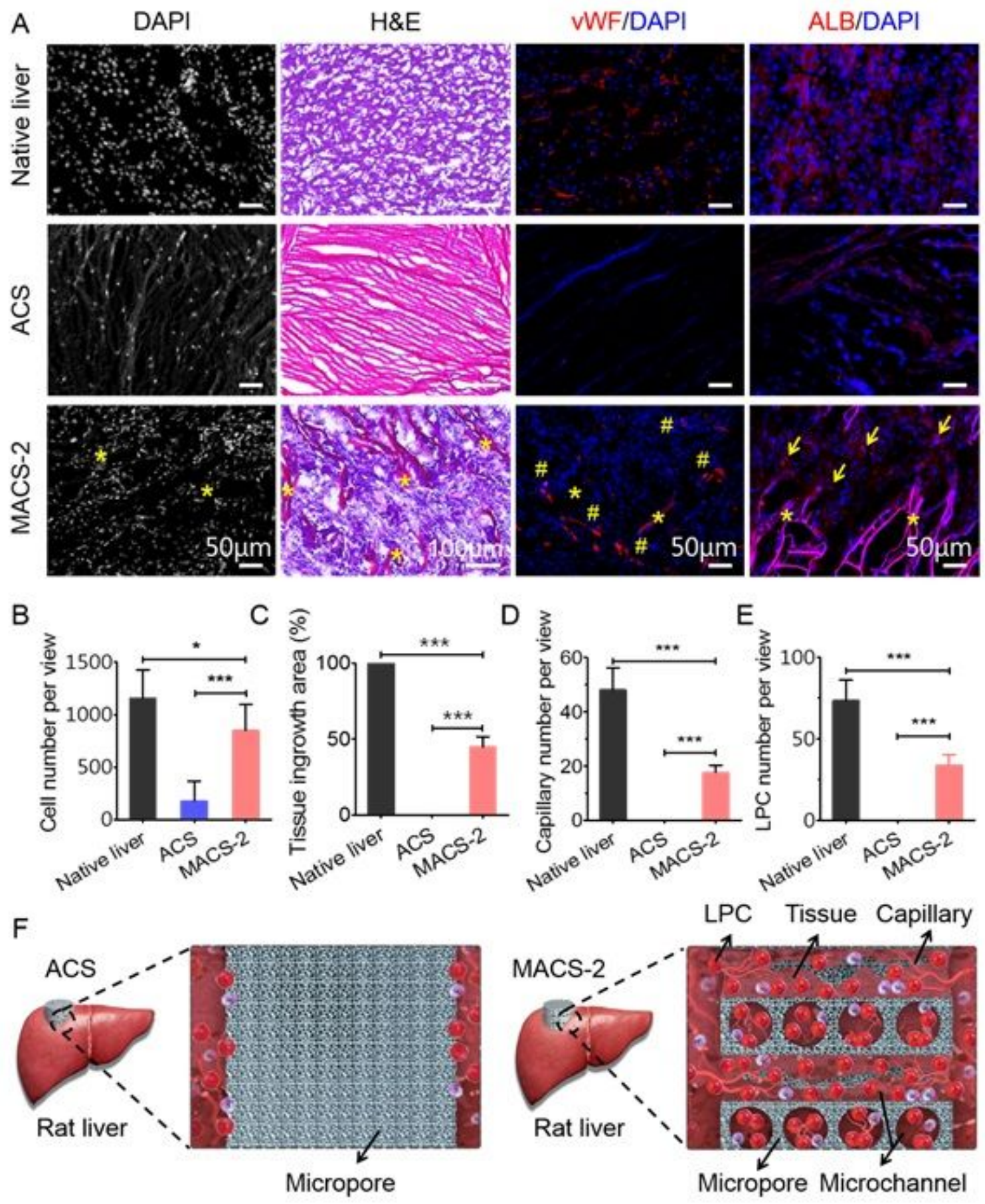

Figure 9

Liver regeneration in rat models after implantation of the ACS and MACS-2. (A) DAPI staining showing cell infiltration within the ACS and MACS-2. H\&E staining showing tissue ingrowth. Yellow asterisk (*) represents the alkylated CS. Images of immunofluorescent staining for VWF (red) and ALB (red) indicating capillary and liver parenchymal cell (LPC) infiltration within the ACS and MACS-2. Yellow 
pound key (\#) and arrow represent capillary and LPC, respectively. (B, C, D, E) Quantification of cell number, tissue ingrowth area, capillary number, and LPCs per view within the ACS and MACS-2. (F) Schematic illustration of in situ liver regeneration, including the host cell infiltration and vascularization. $n=3$, Data are means \pm SD. ns indicated no significant difference, ${ }^{*} P<0.05,{ }^{* *} P<0.01,{ }^{* * *} P<0.001$.

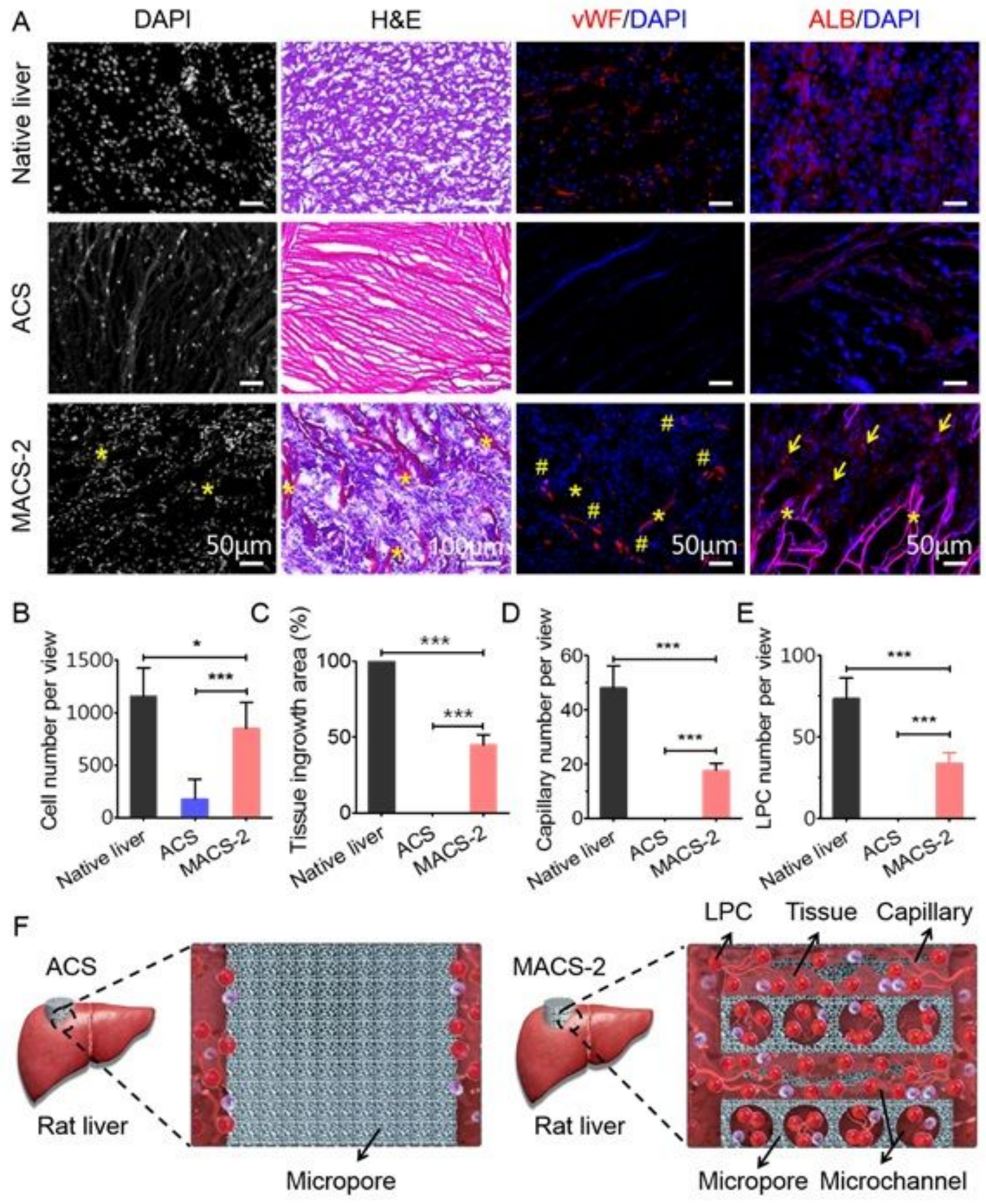

Figure 9

Liver regeneration in rat models after implantation of the ACS and MACS-2. (A) DAPI staining showing cell infiltration within the ACS and MACS-2. H\&E staining showing tissue ingrowth. Yellow asterisk (*) represents the alkylated CS. Images of immunofluorescent staining for VWF (red) and ALB (red) indicating capillary and liver parenchymal cell (LPC) infiltration within the ACS and MACS-2. Yellow 
pound key (\#) and arrow represent capillary and LPC, respectively. (B, C, D, E) Quantification of cell number, tissue ingrowth area, capillary number, and LPCs per view within the ACS and MACS-2. (F) Schematic illustration of in situ liver regeneration, including the host cell infiltration and vascularization. $n=3$, Data are means \pm SD. $n s$ indicated no significant difference, ${ }^{*} P<0.05,{ }^{* *} P<0.01,{ }^{* \star *} P<0.001$.

\section{Supplementary Files}

This is a list of supplementary files associated with this preprint. Click to download.

- SupplementaryMovie1.mp4

- SupplementaryMovie1.mp4

- SupplementaryMovie2.mp4

- SupplementaryMovie2.mp4

- SupplementaryMovie3.mp4

- SupplementaryMovie3.mp4

- SupplementaryMovie4.mp4

- SupplementaryMovie4.mp4

- SupplementaryMovie5.mp4

- SupplementaryMovie5.mp4

- SupplementaryMovie6.mp4

- SupplementaryMovie6.mp4

- SupplementaryMovie7.mp4

- SupplementaryMovie7.mp4

- SupplementaryMovie8.mp4

- SupplementaryMovie8.mp4

- SupplementaryMovie9.mp4

- SupplementaryMovie9.mp4

- SupplementaryMovie10.mp4

- SupplementaryMovie10.mp4

- SupplementaryMovie11.mp4

- SupplementaryMovie11.mp4

- SupplementaryMovie12.mp4

- SupplementaryMovie12.mp4

- SupplementaryMovie13.mp4

- SupplementaryMovie13.mp4

- FigureS1.jpg 
- FigureS1.jpg

- Figures2.jpg

- FigureS2.jpg

- FigureS3.jpg

- FigureS3.jpg

- FigureS4.jpg

- FigureS4.jpg

- FigureS5.jpg

- FigureS5.jpg

- FigureS6.jpg

- FigureS6.jpg

- FigureS7.jpg

- FigureS7.jpg 